\title{
REAL RANK AND TOPOLOGICAL DIMENSION OF HIGHER RANK GRAPH ALGEBRAS
}

\author{
DAVID PASK, ADAM SIERAKOWSKI, AND AIDAN SIMS
}

\begin{abstract}
We study dimension theory for the $C^{*}$-algebras of row-finite $k$-graphs with no sources. We establish that strong aperiodicity - the higher-rank analogue of condition $(\mathrm{K})$ - for a $k$-graph is necessary and sufficient for the associated $C^{*}$-algebra to have topological dimension zero. We prove that a purely infinite 2 -graph algebra has real-rank zero if and only if it has topological dimension zero and satisfies a homological condition that can be characterised in terms of the adjacency matrices of the 2-graph. We also show that a $k$-graph $C^{*}$-algebra with topological dimension zero is purely infinite if and only if all the vertex projections are properly infinite. We show by example that there are strongly purely infinite 2-graphs algebras, both with and without topological dimension zero, that fail to have real-rank zero.
\end{abstract}

\section{INTRODUCTION}

Dimension theory of $C^{*}$-algebras has played an important role in the subject over the last few decades. In particular, notions of dimension such as real rank, stable rank, decomposition rank and nuclear dimension have become more and more prominent in classification theory. But these notions of dimension can be difficult to compute for concrete classes of examples. In this paper we focus on dimension theory of higher rank graph $C^{*}$-algebras. Specifically we consider topological dimension zero and real-rank zero of $C^{*}$-algebras associated to higher rank graphs.

A $C^{*}$-algebra $A$ has topological dimension zero if the primitive ideal space of $A$ endowed with the Jacobsen topology has a basis of compact open sets. This definition can be found in Brown and Pedersen's work [5], but the study of the concept goes back much further. For example Bratteli and Elliott showed [1] that a separable $C^{*}$-algebra $A$ of type I is $\mathrm{AF}$ if and only if it has topological dimension zero. For more recent work on topological dimension see [3, 5, 16, 28, 30, 31, 35, 38].

Recently, sufficient conditions on a $k$-graph $\Lambda$ for its $C^{*}$-algebra to have topological dimension zero were established by Kang and the first named author. They proved in [16, Theorem 4.2] that for a row-finite $k$-graph $\Lambda$ with no sources, the $C^{*}$-algebra associated to $\Lambda$ has topological dimension zero if $\Lambda$ is strongly aperiodic. Strong aperiodicity is the higher-rank analogue of the well-known condition $(K)$ for directed graphs (every vertex that is the basepoint of a first-return path is the basepoint of at least two such paths). It can be characterised combinatorially in terms of the $k$-graph, and can be rephrased in a number of other ways. For example, it is equivalent to the condition on the infinite-path groupoid $\mathcal{G}_{\Lambda}$ of $\Lambda$, that for every closed invariant subset of the unit space, the points with

Date: September 29, 2018.

2010 Mathematics Subject Classification. 46L05.

Key words and phrases. Graph $C^{*}$-algebra; real rank; topological dimension; purely infinite; higherrank graph.

This research was supported by the Australian Research Council. 
trivial isotropy are dense. It is also equivalent to the property that every ideal of the $k$-graph $C^{*}$-algebra $C^{*}(\Lambda)$ is gauge-invariant. We prove in Section 3 that this sufficient condition is also necessary (Theorem 3.2). Our approach uses the fact that topological dimension zero passes to ideals and quotients, together with recent results on $P$-graphs, induced algebras, and primitive-ideal structure of $k$-graph algebras [6, 39]. We finish the section with Corollary 3.9 which describes a number of properties of $\Lambda$ and $C^{*}(\Lambda)$ that are all equivalent to strong aperiodicity of $\Lambda$, and hence topological dimension zero for $C^{*}(\Lambda)$.

We then consider real-rank zero for purely infinite $k$-graph $C^{*}$-algebras. A $C^{*}$-algebra $A$ has real-rank zero if the set of invertible self-adjoint elements of the minimal unitisation of $A$ is dense in the set of all self-adjoint elements in the minimal unitisation of $A[4]$. For $k=1$, it was established in the locally finite case in [14, 15], and in general by Hong and Szymański in [13, Theorem 2.5], that a graph $C^{*}$-algebra $C^{*}(E)$ has real-rank zero if and only if the graph satisfies Condition $(\mathrm{K})$. However, the methods of [14, 15, 13, do not generalise to $k$-graphs. So we restrict our attention to the special case of $k$-graphs $\Lambda$ for which $C^{*}(\Lambda)$ is purely infinite.

Simple purely infinite $C^{*}$-algebras are automatically of real-rank zero by [4, 40, but nonsimple purely infinite $C^{*}$-algebras need not have real-rank zero (see Examples 6.1 and 6.2). Indeed, Pasnicu and Rørdam have proved that a purely infinite $C^{*}$-algebra has realrank zero if and only if it has topological dimension zero and satisfies a $K$-theoretic criterion called $K_{0}$-liftability [31. Using this result we derive a homological condition characterising when a purely infinite 2 -graph $C^{*}$-algebra has real-rank zero. We do this by examining Evans' spectral-sequence calculation of $K$-theory for $k$-graph $C^{*}$-algebras. This sequence is based on a complex $D_{*}^{\Lambda}$ in which the terms are the exterior powers of $\mathbb{Z}^{k}$ tensored with $\mathbb{Z} \Lambda^{0}$. When $k=2$, Evans proves that $K_{1}\left(C^{*}(\Lambda)\right)$ is isomorphic to the first homology group of this complex. We show that the inclusion $j^{H}: H \hookrightarrow \Lambda^{0}$ of a saturated hereditary set $H$ induces a morphism from the spectral sequence for $\Gamma:=H \Lambda$ to the spectral sequence for $\Lambda$. Using naturality of Kasparov's spectral sequence, we show that for $k=2$ the map in $K_{1}$ induced by the inclusion $C^{*}(\Gamma) \hookrightarrow C^{*}(\Lambda)$ coincides with the map $H_{1}\left(j^{H}\right):=H_{1}\left(D_{*}^{\Gamma}\right) \rightarrow H_{1}\left(D_{*}^{\Gamma}\right)$ induced by the inclusion $H \hookrightarrow \Lambda^{0}$. Combining this with Pasnicu and Rørdam's result yields our characterisation of real-rank zero: a purely infinite 2-graph $C^{*}$-algebra has real rank zero if and only if $\Lambda$ is strongly aperiodic and $H_{1}\left(j^{H}\right)$ is injective for each saturated hereditary subset $H$ of $\Lambda^{0}$ (Theorem 4.3). Both of these conditions are necessary for $C^{*}(\Lambda)$ to have real rank zero even when $C^{*}(\Lambda)$ is not purely infinite. Moreover, the injectivity of each $H_{1}\left(j^{H}\right)$ can be reformulated as an elementary algebraic condition involving the adjacency matrices of the ambient 2-graph.

Our resulting condition on $\Lambda$ is much more subtle than the corresponding result for 1 -graphs, reflecting the more nuanced $K$-theory of $k$-graph $C^{*}$-algebras in which, for example, the $K_{0^{-}}$classes of vertex projections do not necessarily generate the whole $K_{0^{-}}$ group (see [10]).

Since pure infiniteness is a hypothesis in Theorem 4.3, we develop in Section 5 a characterisation of pure infiniteness for the $C^{*}$-algebras of strongly aperiodic $k$-graphs. This topic has been intensively studied, and for 1-graphs this culminated in [13], where sufficient and necessary conditions for pure infiniteness of a 1-graph $C^{*}$-algebra (in terms of the 1-graph) where established. Building on recent results for cofinal $k$-graphs in [2], we establish a number of properties equivalent to pure infiniteness of $C^{*}(\Lambda)$. In particular, generalising [2, Corollary 5.1], we prove that $C^{*}(\Lambda)$ is purely infinite if and only if the 
vertex projections $\left\{s_{v}: v \in \Lambda^{0}\right\}$ are all properly infinite (without assuming $\Lambda$ is cofinal). We also show that this is equivalent to asking that for any saturated hereditary $H \subseteq \Lambda^{0}$, the vertex projections of $C^{*}(\Lambda \backslash \Lambda H)$ are all infinite (not, a priori, properly infinite).

Using this, we prove that if a $k$-graph has an aperiodic quartet [16] at every vertex, then its $C^{*}$-algebra is purely infinite (Proposition 5.1). Our result adds to the list (see [11, 21, 37]) of known sufficient conditions on a $k$-graph $\Lambda$ for pure infiniteness of its $C^{*}$-algebra. The utility of our result becomes apparent in Section 6, where we use Proposition 5.1 to construct two examples of purely infinite $k$-graph $C^{*}$-algebras without real-rank zero. The first example is a 2-graph $\Lambda$ such that $C^{*}(\Lambda)$ is purely infinite and has topological dimension zero, but fails to have real-rank zero because the morphism $H_{1}\left(j^{H}\right)$, for a suitable $H \subseteq \Lambda^{0}$, is not injective. Our second example is also purely infinite, but fails to have real-rank zero because the graph is not strongly aperiodic, and so its $C^{*}$-algebra does not have topological dimension zero. Finally we present an example of a 2-graph which is both strongly aperiodic and satisfies the injectivity condition, but whose $C^{*}$-algebra is not purely infinite, and fails to have real rank zero.

\section{Preliminaries AND NOtATion}

We let $\operatorname{Prim}(A)$ denote the set of all primitive ideals in a $C^{*}$-algebra $A$. The Jacobsen topology on $\operatorname{Prim}(A)$ is defined as follows. For each ideal $I$ of $A$, we define the hull of $I$ by $\operatorname{hull}(I):=\{P \in \operatorname{Prim}(A): I \subseteq P\}$; and for each subset $S \subseteq \operatorname{Prim}(A)$, we define the kernel of $S$ to be $\operatorname{ker}(S):=\bigcap_{P \in S} P$. The Jacobsen topology is then determined by the closure operation: $\bar{S}:=\operatorname{hull}(\operatorname{ker}(S))$ for every $S \subseteq \operatorname{Prim}(A)$.

Following [21, 25, 32] we briefly recall the notion of a $k$-graph and the associated notation. For $k \geq 0$, a $k$-graph is a nonempty countable small category equipped with a functor $d: \Lambda \rightarrow \mathbb{N}^{k}$ that satisfies the factorisation property: for all $\lambda \in \Lambda$ and $m, n \in \mathbb{N}^{k}$ such that $d(\lambda)=m+n$ there exist unique $\mu, \nu \in \Lambda$ such that $d(\mu)=m, d(\nu)=n$, and $\lambda=\mu \nu$. When $d(\lambda)=n$ we say $\lambda$ has degree $n$, and we write $\Lambda^{n}=d^{-1}(n)$. The standard generators of $\mathbb{N}^{k}$ are denoted $e_{1}, \ldots, e_{k}$, and we write $n_{i}$ for the $i^{\text {th }}$ coordinate of $n \in \mathbb{N}^{k}$. For $m, n \in \mathbb{N}^{k}$, we write $m \vee n$ for their coordinate-wise maximum, and define a partial order on $\mathbb{N}^{k}$ by $m \leq n$ if $m_{i} \leq n_{i}$ for all $i$.

If $\Lambda$ is a $k$-graph, its vertices are the elements of $\Lambda^{0}$. The factorisation property implies that these are precisely the identity morphisms, and so can be identified with the objects. For $\alpha \in \Lambda$ the source $s(\alpha)$ is the domain of $\alpha$, and the range $r(\alpha)$ is the codomain of $\alpha$ (strictly speaking, $s(\alpha)$ and $r(\alpha)$ are the identity morphisms associated to the domain and codomain of $\alpha$ ). Given $\lambda, \mu \in \Lambda$ and $E \subseteq \Lambda$, we define

$$
\begin{gathered}
\lambda E=\{\lambda \nu: \nu \in E, r(\nu)=s(\lambda)\}, \quad E \mu=\{\nu \mu: \nu \in E, s(\nu)=r(\mu)\}, \quad \text { and } \\
\lambda E \mu=\lambda E \cap E \mu .
\end{gathered}
$$

For $X, E, Y \subseteq \Lambda$, we write $X E Y$ for $\bigcup_{\lambda \in X, \mu \in Y} \lambda E \mu$. We say the $k$-graph $\Lambda$ is row-finite if the set $v \Lambda^{m}$ is finite for each $m \in \mathbb{N}^{k}$ and $v \in \Lambda^{0}$. Also, $\Lambda$ has no sources if $v \Lambda^{e_{i}} \neq \emptyset$ for all $v \in \Lambda^{0}$ and $i \in\{1, \ldots, k\}$.

For $\lambda \in \Lambda$ and $0 \leq m \leq n \leq d(\lambda)$, we write $\lambda(m, n)$ for the unique path in $\Lambda$ such that $\lambda=\lambda^{\prime} \lambda(m, n) \lambda^{\prime \prime}$, where $d\left(\lambda^{\prime}\right)=m, d(\lambda(m, n))=n-m$ and $d\left(\lambda^{\prime \prime}\right)=d(\lambda)-n$. We write $\lambda(n)$ for $\lambda(n, n)=s(\lambda(0, n))$. We denote by $\Omega_{k}$ the $k$-graph with vertices $\Omega_{k}^{0}:=\mathbb{N}^{k}$, paths $\Omega_{k}^{m}:=\left\{(n, n+m), n \in \mathbb{N}^{k}\right\}$ for $m \in \mathbb{N}^{k}, r((n, n+m))=n$ and $s((n, n+m))=n+m$. 
Let $\Lambda$ be a row-finite $k$-graph with no sources. A Cuntz-Krieger $\Lambda$-family in a $C^{*}$ algebra $B$ is a function $s: \lambda \mapsto s_{\lambda}$ from $\Lambda$ to $B$ such that

(CK1) $\left\{s_{v}: v \in \Lambda^{0}\right\}$ is a collection of mutually orthogonal projections;

(CK2) $s_{\mu} s_{\nu}=s_{\mu \nu}$ whenever $s(\mu)=r(\nu)$;

(CK3) $s_{\lambda}^{*} s_{\lambda}=s_{s(\lambda)}$ for all $\lambda \in \Lambda$; and

(CK4) $s_{v}=\sum_{\lambda \in v \Lambda^{n}} s_{\lambda} s_{\lambda}^{*}$ for all $v \in \Lambda^{0}$ and $n \in \mathbb{N}^{k}$.

The $k$-graph $C^{*}$-algebra $C^{*}(\Lambda)$ is the universal $C^{*}$-algebra generated by a Cuntz-Krieger $\Lambda$-family. For more details see [21].

\section{TOPOLOGICAL Dimension ZERO}

In this section we study when $k$-graph $C^{*}$-algebras have topological dimension zero. This requires some machinery, which we now introduce. Let $\Lambda$ be a row-finite $k$-graph with no sources. A subset $H$ of $\Lambda^{0}$ is hereditary if $s(H \Lambda) \subseteq H$. We say $H \subseteq \Lambda^{0}$ is saturated if for all $v \in \Lambda^{0}$

$$
\left\{s(\lambda): \lambda \in v \Lambda^{e_{i}}\right\} \subseteq H \text { for some } i \in\{1, \ldots, k\} \quad \Longrightarrow \quad v \in H .
$$

(Since $\Lambda$ has no sources, we do not need to worry about whether $r^{-1}(v)$ is empty - cf. [32].) For a hereditary $H \subseteq \Lambda^{0}$ we write $I_{H}$ for the ideal in $C^{*}(\Lambda)$ generated by $\left\{s_{v}: v \in H\right\}$. If $H$ is hereditary, then $H \Lambda$ is a row-finite $k$-graph with no sources, and if $H$ is also saturated, then $\Lambda \backslash \Lambda H$ is also a row-finite $k$-graph with no sources (see [21]).

Let $\Lambda$ be a row-finite $k$-graph with no sources. The set

$$
\Lambda^{\infty}:=\left\{x: \Omega_{k} \rightarrow \Lambda \mid x \text { is a degree-preserving functor }\right\}
$$

is called the infinite-path space of $\Lambda$. For $v \in \Lambda^{0}$, we write $v \Lambda^{\infty}:=\left\{x \in \Lambda^{\infty}: x(0)=v\right\}$, and for $x \in \Lambda^{\infty}$, we write $r(x):=x(0) \in \Lambda^{0}$. For $p \in \mathbb{N}^{k}$, the shift map $\sigma^{p}: \Lambda^{\infty} \rightarrow \Lambda^{\infty}$ defined by $\left(\sigma^{p} x\right)(m, n)=x(m+p, n+p)$ for $(m, n) \in \Omega_{k}$ is a local homeomorphism. For $\lambda \in \Lambda$ and $x \in \Lambda^{\infty}$ with $s(\lambda)=r(x)$ we write $\lambda x$ for the unique element $y \in \Lambda^{\infty}$ such that $\lambda=y(0, d(\lambda))$ and $x=\sigma^{d(\lambda)} y$, see [21].

Definition 3.1. Let $\Lambda$ be a row-finite $k$-graph with no sources. We say that $\Lambda$ is aperiodic (or satisfies the aperiodicity condition) if for every vertex $v \in \Lambda^{0}$ there exist an infinite path $x \in v \Lambda^{\infty}$ such that $\sigma^{m}(x) \neq \sigma^{n}(x)$ for all $m \neq n \in \mathbb{N}^{k}$, see [21, 32, 36]. If $\Lambda \backslash \Lambda H$ is aperiodic for every hereditary saturated $H \subsetneq \Lambda^{0}$, we say $\Lambda$ is strongly aperiodic, see [16].

We can now state our main result about topological dimension of $k$-graph $C^{*}$-algebras. Let us emphasise that half of the hard work has already been done for us: The implication (ii) $\Rightarrow$ (i) was established in [16] by Kang and the first named author. As we shall see the reverse implication (i) $\Rightarrow$ (ii) also requires some non-trivial work.

Theorem 3.2. Let $\Lambda$ be a row-finite $k$-graph with no sources. Then the following are equivalent:

(i) The $C^{*}$-algebra $C^{*}(\Lambda)$ has topological dimension zero.

(ii) The $k$-graph $\Lambda$ is strongly aperiodic.

Before we prove Theorem 3.2 we present six lemmata. The key ideas, namely the use of $P$-graphs and induced algebras, are introduced in Lemmas 3.3 and 3.4 . We briefly recall the notation involved.

Following [16] we recall the definition of a maximal tail. Let $\Lambda$ be a row-finite $k$-graph with no sources. A nonempty subset $T$ of $\Lambda^{0}$ is called a maximal tail if 
(a) for all $v_{1}, v_{2} \in T$ there exists $w \in T$ such that $v_{1} \Lambda w \neq \emptyset$ and $v_{2} \Lambda w \neq \emptyset$,

(b) for every $v \in T$ and $1 \leq i \leq k$ there exist $e \in v \Lambda^{e_{i}}$ such that $s(e) \in T$, and

(c) for all $w \in T$ and $v \in \Lambda^{0}$ with $v \Lambda w \neq \emptyset$ we have $v \in T$.

Following [6] we recall the definition of $\operatorname{Per}(\Lambda)$ and $H_{\text {Per }}$. Let $\Lambda$ be a row-finite $k$-graph with no sources such that $\Lambda^{0}$ is a maximal tail. Define a relation $\sim$ on $\Lambda$ by $\mu \sim \nu$ if and only if $s(\mu)=s(\nu)$ and $\mu x=\nu x$ for all $x \in s(\mu) \Lambda^{\infty}$. Then [6, Lemma 4.2(1)] says that the set $\operatorname{Per}(\Lambda):=\{d(\mu)-d(\nu): \mu, \nu \in \Lambda$ and $\mu \sim \nu\}$ is a subgroup of $\mathbb{Z}^{k}$, called the periodicity group of $\Lambda$. Moreover, it follows from [6, Lemma 4.6], that

$\operatorname{Per}(\Lambda)=\left\{l \in \mathbb{Z}^{k}:\right.$ there exists $w \in \Lambda^{0}$ and $n, m \in \mathbb{N}^{k}$ such that $l=n-m$ and $\sigma^{n}(y)=\sigma^{m}(y)$ for all $\left.y \in w \Lambda^{\infty}\right\}$.

Let

$H_{\text {Per }}:=\left\{v \in \Lambda^{0}:\right.$ for all $\lambda \in v \Lambda$ and $m \in \mathbb{N}^{k}$ such that $d(\lambda)-m \in \operatorname{Per}(\Lambda)$, there exists $\mu \in v \Lambda^{m}$ such that $\left.\lambda \sim \mu\right\}$.

Lemma 4.2 of [6] shows that $H_{\mathrm{Per}}$ is a non-empty hereditary set (not always saturated).

We will also need to use the $P$-graphs which were introduced in [6]. The definitions of $P$-graphs, where $P$ is a finitely generated cancellative abelian monoid, and the associated $C^{*}$-algebras are obtained by replacing $\mathbb{N}^{k}$ with $P$ in the definitions for $k$-graphs given above, see [6, Section 2] for details. If $\Gamma$ is a $P$-graph we will continue to call a family satisfying (CK1)-(CK4) for $\Gamma$ with $P$ replacing $\mathbb{N}^{k}$ a Cuntz-Krieger $\Gamma$-family. If

$$
P:=\left\{m+\operatorname{Per}(\Lambda): m \in \mathbb{N}^{k}\right\} \subseteq \mathbb{Z}^{k} / \operatorname{Per}(\Lambda),
$$

then $\left(H_{\mathrm{Per}} \Lambda\right) / \sim$ is a $P$-graph with operations inherited from $\Lambda$ and degree map given by $\tilde{d}([\lambda])=d(\lambda)+\operatorname{Per}(\Lambda)$.

Lemma 3.3. Let $\Lambda$ be a row-finite $k$-graph with no sources such that $\Lambda^{0}$ is a maximal tail. Define $H:=H_{\text {Per }}$ and let $\Gamma:=(H \Lambda) / \sim$ be the P-graph described above. There exist an action $\alpha$ of the annihilator $\operatorname{Per}(\Lambda)^{\perp}:=\left\{h \in \operatorname{Hom}\left(\mathbb{Z}^{k}, \mathbb{T}\right):\left.h\right|_{\operatorname{Per}(\Lambda)}=1\right\}$ on $C^{*}(\Gamma)$ such that

$$
\alpha_{h}\left(s_{[\lambda]}\right)=h(d(\lambda))^{-1} s_{[\lambda]}, \quad \text { for all } \quad h \in \operatorname{Per}(\Lambda)^{\perp} \text { and } \lambda \in H \Lambda .
$$

Proof. Fix $h \in \operatorname{Per}(\Lambda)^{\perp}$ and $\lambda, \mu \in H \Lambda$ such that $[\lambda]=[\mu]$. We prove that $h(d(\lambda))=$ $h(d(\mu))$. Since $\lambda \sim \mu$ we have $s(\lambda)=s(\mu)$ and $\lambda x=\mu x$ for all $x \in s(\lambda)(H \Lambda)^{\infty}$. Set $w=s(\lambda) \in H$ (since $H$ is hereditary). For any $x \in w \Lambda^{\infty}$ we have $x \in s(\lambda)(H \Lambda)^{\infty}$ so $\sigma^{d(\lambda)}(x)=\sigma^{d(\lambda)+d(\mu)}(\mu x)=\sigma^{d(\lambda)+d(\mu)}(\lambda x)=\sigma^{d(\mu)}(x)$. In particular $d(\lambda)-d(\mu) \in \operatorname{Per}(\Lambda)$. Since $h \in \operatorname{Per}(\Lambda)^{\perp}$ we see that $h(d(\lambda)-d(\mu))=1$, so $h(d(\lambda))=h(d(\mu))$.

Fix $h \in \operatorname{Per}(\Lambda)^{\perp}$. By the preceding paragraph, for $[\lambda] \in \Gamma$, we can define

$$
t_{[\lambda]}:=h(d(\lambda))^{-1} s_{[\lambda]} .
$$

Let $P:=\left\{m+\operatorname{Per}(\Lambda): m \in \mathbb{N}^{k}\right\} \subseteq \mathbb{Z}^{k} / \operatorname{Per}(\Lambda)$. Since $\Gamma$ is a $P$-graph and $C^{*}(\Gamma)$ is the universal algebra generated by a Cuntz-Krieger $\Gamma$-family $\left\{s_{[\lambda]}:[\lambda] \in \Gamma=(H \Lambda) / \sim\right\}$, the set $\left\{t_{[\lambda]}:[\lambda] \in \Gamma\right\}$ is also a Cuntz-Krieger $\Gamma$-family. So the universal property yields a homomorphism $\alpha_{h}: C^{*}(\Gamma) \rightarrow C^{*}(\Gamma)$ such that $\alpha_{h}\left(s_{[\lambda]}\right)=t_{[\lambda]}=h(d(\lambda))^{-1} s_{[\lambda]}$.

For each $g, h \in \operatorname{Per}(\Lambda)^{\perp}, \alpha_{h g}=\alpha_{h} \circ \alpha_{g}$ and $\alpha_{h h^{-1}}=\operatorname{id}_{C^{*}(\Gamma)}$ on the generators of $C^{*}(\Gamma)$, and hence on all of $C^{*}(\Gamma)$. It follows that $h \mapsto \alpha_{h}$ is a group homomorphism from $\operatorname{Per}(\Lambda)^{\perp}$ into the automorphisms of $C^{*}(\Gamma)$. 
Finally we show $h \mapsto \alpha_{h}(a)$ is continuous for each $a \in C^{*}(\Gamma)$. Let $a=s_{[\lambda]}$ for some $\lambda \in H \Lambda$ and suppose $h_{i} \rightarrow h$ in $\operatorname{Per}(\Lambda)^{\perp}$. By definition of the topology on $\operatorname{Hom}\left(\mathbb{Z}^{k}, \mathbb{T}\right)$ we have $h_{i}(d(\lambda)) \rightarrow h(d(\lambda))$ in $\mathbb{T}$, and so $\alpha_{h_{i}}(a) \rightarrow \alpha_{h}(a)$ in $C^{*}(\Gamma)$. Since addition is continuous, we see that $\alpha_{h_{i}}(a) \rightarrow \alpha_{h}(a)$ for $a \in \operatorname{span}\left\{t_{\eta} t_{\nu}^{*}: \eta, \nu \in \Gamma\right\}$; an $\varepsilon / 3$-argument then gives continuity for each $a \in C^{*}(\Gamma)$.

Following [39, p. 100] let $X$ be a right $G$-space and $(A, G, \alpha)$ a dynamical system. If $f: X \rightarrow A$ is a continuous function such that

$$
f(x \cdot s)=\alpha_{s}^{-1}(f(x)), \quad \text { for all } x \in X \text { and } s \in G
$$

then $x \mapsto\|f(x)\|$ is continuous on the orbit space $X / G:=\{x \cdot G: x \in X\}$. The induced algebra is

$$
\operatorname{Ind}_{G}^{X}(A, \alpha)=\left\{f \in C_{b}(X, A): f \text { satisfies (3.1) and } x \cdot G \mapsto\|f(x)\| \text { is in } C_{0}(X / G)\right\} \text {. }
$$

For $n \in \mathbb{N}^{k}$ and $z \in \mathbb{T}^{k}$, we write $z^{n}$ for $\prod_{i=1}^{k} z_{i}^{n_{i}}$.

In what follows we identify $\operatorname{Per}(\Lambda)^{\perp}$ with $\left\{z \in \mathbb{T}^{k}: z^{n}=1\right.$ for all $\left.n \in \operatorname{Per}(\Lambda)\right\} \subseteq \mathbb{T}^{k}$. Since $\operatorname{Per}(\Lambda)^{\perp}$ acts on $\mathbb{T}^{k}$ by right multiplication $z \cdot w:=z w$ [39, Example 3.34], $\mathbb{T}^{k}$ is a right $\operatorname{Per}(\Lambda)^{\perp}$-space. So, using the action $\alpha: \operatorname{Per}(\Lambda)^{\perp} \rightarrow \operatorname{Aut}\left(C^{*}(\Gamma)\right)$ of Lemma 3.3, we can form the induced algebra $\operatorname{Ind}(\alpha):=\operatorname{Ind}_{\operatorname{Per}(\Lambda)^{\perp}}^{\mathbb{T}^{k}}\left(C^{*}(\Gamma), \alpha\right)$.

Recall that if $\Lambda$ is a row-finite $k$-graph with no sources, then the universal property of $C^{*}(\Lambda)$ ensures that it carries a canonical action $\gamma$, called the gauge action, of $\mathbb{T}^{k}$ satisfying $\gamma_{z}\left(s_{\lambda}\right)=z^{d(\lambda)} s_{\lambda}($ see [21]).

Lemma 3.4. Let $\Lambda, H, \alpha$ and $\Gamma$ be as in Lemma 3.3. Then there exists an isomorphism $\pi: C^{*}(H \Lambda) \rightarrow \operatorname{Ind}(\alpha)$ such that $\pi\left(s_{\lambda}\right)(z)=z^{d(\lambda)} s_{[\lambda]}$ for each $\lambda \in H \Lambda$ and $z \in \mathbb{T}^{k}$. We have

$$
\operatorname{Prim}\left(C^{*}(H \Lambda)\right) \cong\left(\mathbb{T}^{k} \times \operatorname{Prim}\left(C^{*}(\Gamma)\right)\right) / \operatorname{Per}(\Lambda)^{\perp}
$$

where the action of $\operatorname{Per}(\Lambda)^{\perp}$ on $\mathbb{T}^{k} \times \operatorname{Prim}\left(C^{*}(\Gamma)\right)$ is given by $(z, I) \cdot w=\left(z w, \alpha_{w^{-1}}(I)\right)$.

Proof. For each $\lambda \in H \Lambda$ define $t_{\lambda}: \mathbb{T}^{k} \rightarrow C^{*}(\Gamma)$ by $t_{\lambda}(z)=z^{d(\lambda)} s_{[\lambda]}$. We show that each $t_{\lambda}$ belongs to the $C^{*}$-algebra $\operatorname{Ind}(\alpha)$ described above.

For fixed $\lambda \in H \Lambda, z \mapsto z^{d(\lambda)}$ is continuous from $\mathbb{T}^{k}$ to $\mathbb{T}$, so $t_{\lambda}$ belongs to $C\left(\mathbb{T}^{k}, C^{*}(\Gamma)\right)$. Fix $z \in \mathbb{T}^{k}$ and $w \in \operatorname{Per}(\Lambda)^{\perp}$. We have

$$
\begin{aligned}
\alpha_{w}^{-1}\left(t_{\lambda}(z)\right) & =\alpha_{w}^{-1}\left(z^{d(\lambda)} s_{[\lambda]}\right)=z^{d(\lambda)} \alpha_{w^{-1}}\left(s_{[\lambda]}\right) \\
& =z^{d(\lambda)} w^{d(\lambda)} s_{[\lambda]}=(z w)^{d(\lambda)} s_{[\lambda]}=t_{\lambda}(z w)=t_{\lambda}(z \cdot w) .
\end{aligned}
$$

So $t_{\lambda}$ satisfies (3.1). The function on $\mathbb{T}^{k} / \operatorname{Per}(\Lambda)^{\perp}$ given by $z \cdot \operatorname{Per}(\Lambda)^{\perp} \mapsto\left\|t_{\lambda}(z)\right\|$ is constant, hence continuous. Since $\mathbb{T}^{k} / \operatorname{Per}(\Lambda)^{\perp}$ is compact and Hausdorff (see [39, p. 101]), the map $z \cdot \operatorname{Per}(\Lambda)^{\perp} \mapsto\left\|t_{\lambda}(z)\right\|$ belongs to $C_{0}\left(\mathbb{T}^{k} / \operatorname{Per}(\Lambda)^{\perp}\right)$, so $t_{\lambda} \in \operatorname{Ind}(\alpha)$.

Since $H \Lambda$ is a $k$-graph and $C^{*}(H \Lambda)$ is the universal algebra generated by the CuntzKrieger family $\left\{s_{\lambda}: \lambda \in H \Lambda\right\}$ it easily follows that $\left\{t_{\lambda}: \lambda \in H \Lambda\right\}$ satisfies (CK1)-(CK3). To verify (CK4) fix $v \in H$ and $n \in \mathbb{N}^{k}$, let $p:=n+\operatorname{Per}(\Lambda)$, and define $\varphi: v \Lambda^{n} \rightarrow v \Gamma^{p}$, by $\varphi(\lambda)=[\lambda]$. It suffices to show that $\varphi$ is a bijection. To check that $\varphi$ is surjective (this is non-trivial because $\left.[\lambda] \in v \Gamma^{p} \nRightarrow d(\lambda)=n\right)$, choose $[\lambda] \in v \Gamma^{p}$. Since $\tilde{d}([\lambda])=d(\lambda)+\operatorname{Per}(\Lambda)$ there exists $\mu \sim \lambda$ such that $d(\mu)=n$ and $r(\mu)=r(\lambda)$ (see [6, Theorem 4.2]). We obtain $\mu \in v \Lambda^{n}$ and $\varphi(\mu)=[\lambda]$. To check that $\varphi$ is injective, suppose that $\varphi(\lambda)=\varphi(\mu)$ for some 
$\lambda, \mu \in v \Lambda^{n}$. Since $\lambda \sim \mu$ and $d(\lambda)=d(\mu)$ it follows from [6, Theorem 4.2] that $\lambda=\mu$. So $\varphi$ is a bijection as claimed.

By the universal property of $C^{*}(H \Lambda)$ there exists a homomorphism $\pi: C^{*}(H \Lambda) \rightarrow$ $\operatorname{Ind}(\alpha)$ such that

$$
\pi\left(s_{\lambda}\right)=t_{\lambda}=\left(z \mapsto z^{d(\lambda)} s_{[\lambda]}\right), \quad \text { for each } \lambda \in H \Lambda
$$

We now show $\pi$ is injective. Similarly to [39, Lemma 3.48], we have a dynamical system $\left(\operatorname{Ind}(\alpha), \mathbb{T}^{k}\right.$, lt $)$ where $\operatorname{lt}_{z}(f)(x)=f(x z)$ for all $z \in \mathbb{T}^{k}$ and $f \in \operatorname{Ind}(\alpha)$. Writing $\gamma$ for the gauge action on $C^{*}(H \Lambda)$, for $x, z \in \mathbb{T}^{k}$ and $\lambda \in H \Lambda$, we have

$$
\begin{aligned}
\operatorname{lt}_{z} \circ \pi\left(s_{\lambda}\right)(x) & =\operatorname{lt}_{z}\left(t_{\lambda}\right)(x)=t_{\lambda}(x z)=(x z)^{d(\lambda)} s_{[\lambda]}=z^{d(\lambda)} x^{d(\lambda)} s_{[\lambda]} \\
& =z^{d(\lambda)} t_{\lambda}(x)=z^{d(\lambda)} \pi\left(s_{\lambda}\right)(x)=\pi\left(z^{d(\lambda)} s_{\lambda}\right)(x)=\pi \circ \gamma_{z}\left(s_{\lambda}\right)(x) .
\end{aligned}
$$

So the homomorphisms $\operatorname{lt}_{z} \circ \pi$ and $\pi \circ \gamma_{z}$ agree on generators, and hence are equal. Since each $t_{\lambda}$ is non-zero it follows from the gauge-invariant uniqueness theorem [21, Theorem 3.4] that $\pi$ is injective.

We now show that $\pi$ is surjective. By [39, Proposition 3.49], $\operatorname{Ind}(\alpha)$ can be regarded as a $C_{0}\left(\mathbb{T}^{k} / \operatorname{Per}(\Lambda)^{\perp}\right)$-algebra in the sense of [39], where

$$
\varphi \cdot f(z)=\varphi\left(z \cdot \operatorname{Per}(\Lambda)^{\perp}\right) f(z)
$$

for all $\varphi \in C_{0}\left(\mathbb{T}^{k} / \operatorname{Per}(\Lambda)^{\perp}\right), f \in \operatorname{Ind}(\alpha)$ and $z \in \mathbb{T}^{k}$. In particular it follows from [39, Proposition C.24 and Theorem C.26] that $\pi$ is surjective provided the following two properties hold:

(i) If $f \in \operatorname{im}(\pi)$ and $\varphi \in C_{0}\left(\mathbb{T}^{k} / \operatorname{Per}(\Lambda)^{\perp}\right)$, then $\varphi \cdot f \in \operatorname{im}(\pi)$.

(ii) For each $z \in \mathbb{T}^{k},\{f(z): f \in \operatorname{im}(\pi)\}$ is dense in $C^{*}(\Gamma)$.

To prove (i), observe that for $n \in \operatorname{Per}(\Lambda)$, the map $z \mapsto z^{n}$ vanishes on $\operatorname{Per}(\Lambda)^{\perp}$, and so descends to an element $\varepsilon_{n}$ of $C_{0}\left(\mathbb{T}^{k} / \operatorname{Per}(\Lambda)^{\perp}\right)$ satisfying

$$
\varepsilon_{n}\left(z \cdot \operatorname{Per}(\Lambda)^{\perp}\right)=z^{n}
$$

The set $\left\{\varepsilon_{n}: n \in \operatorname{Per}(\Lambda)\right\}$ generates $C_{0}\left(\mathbb{T}^{k} / \operatorname{Per}(\Lambda)^{\perp}\right)$ as a $C^{*}$-algebra. So (i) follows once we prove that $\operatorname{im}(\pi)$ is invariant for the action on every $\varepsilon_{n}$. For $f \in \operatorname{Ind}(\alpha)$ and $z \in \mathbb{T}^{k}$, equations (3.3) and (3.4) give

$$
\left(\varepsilon_{n} \cdot f\right)(z)=\varepsilon_{n}\left(z \cdot \operatorname{Per}(\Lambda)^{\perp}\right) f(z)=z^{n} f(z) .
$$

Fix $n \in \operatorname{Per}(\Lambda)$ and $f \in \operatorname{im}(\pi)$. We show that the function $z \mapsto z^{n} f(z)$ belongs to $\operatorname{im}(\pi)$. Write $n=p-q$ with $p, q \in \mathbb{N}^{k}$ and fix $\lambda, \mu \in H \Lambda$. By [6, Theorem 4.2(3)] there exist a bijection $\theta: s(\lambda) \Lambda^{p} \rightarrow s(\lambda) \Lambda^{q}$ such that $\nu \sim \theta(\nu)$ for all $\nu \in s(\lambda) \Lambda^{p}$. Since $\left\{s_{[\lambda]}:[\lambda] \in \Gamma\right\}$ 
satisfies (CK4) and the map $\nu \mapsto[\nu]$ is a bijection from $s(\lambda) \Lambda^{p}$ to $s(\lambda) \Gamma^{p+\operatorname{Per}(\Lambda)}$ we have,

$$
\begin{aligned}
t_{s(\lambda)}(z) & =z^{d(s(\lambda))} s_{[s(\lambda)]}=s_{[s(\lambda)]}=\sum_{[\nu] \in s(\lambda) \Gamma^{p+\operatorname{Per}(\Lambda)}} s_{[\nu]} s_{[\nu]}^{*} \\
& =\sum_{\nu \in s(\lambda) \Lambda^{p}} s_{[\nu]} s_{[\nu]}^{*}=\sum_{\nu \in s(\lambda) \Lambda^{p}} s_{[\nu]} s_{[\theta(\nu)]}^{*} \\
& =\sum_{\nu \in s(\lambda) \Lambda^{p}} z^{-d(\nu)} t_{\nu}(z)\left(z^{-d(\theta(\nu))} t_{\theta(\nu)}(z)\right)^{*} \\
& =z^{-(p-q)} \sum_{\nu \in s(\lambda) \Lambda^{p}} t_{\nu} t_{\theta(\nu)}^{*}(z) .
\end{aligned}
$$

Hence $\left(z \mapsto z^{n} t_{\lambda} t_{\mu}^{*}(z)\right)=\left(z \mapsto\left(\sum_{\nu \in s(\lambda) \Lambda^{p}} t_{\lambda} t_{\nu} t_{\theta(\nu)}^{*} t_{\mu}^{*}\right)(z)\right) \in \operatorname{im}(\pi)$. Finally using that $f \in \overline{\operatorname{span}}\left\{t_{\lambda} t_{\mu}^{*}: \lambda, \mu \in H \Lambda\right\}$ we obtain $\left(z \mapsto z^{n} f(z)\right) \in \operatorname{im}(\pi)$.

To prove (ii), fix $z \in \mathbb{T}^{k}$. For each $\lambda \in H \Lambda$ define $f_{\lambda}:=z^{-d(\lambda)} t_{\lambda} \in \operatorname{im}(\pi)$. Using (3.2) we get $s_{[\lambda]}=f_{\lambda}(z)$, so $s_{[\lambda]} \in\{f(z): f \in \operatorname{im}(\pi)\}$. Since $\operatorname{im}(\pi)$ is a $C^{*}$-algebra and since $\overline{\operatorname{span}}\left\{f_{\lambda} f_{\mu}^{*}(z): \lambda, \mu \in H \Lambda\right\}=C^{*}(\Gamma)$, the set $\{f(z): f \in \operatorname{im}(\pi)\}$ is dense in $C^{*}(\Gamma)$. We conclude that $C^{*}(H \Lambda) \cong \operatorname{Ind}(\alpha)$.

By [39, p. 100], the primitive ideal space of the induced algebra $\operatorname{Ind}(\alpha)$ is homeomorphic with the orbit space $\left(\mathbb{T}^{k} \times \operatorname{Prim}\left(C^{*}(\Gamma)\right)\right) / \operatorname{Per}(\Lambda)^{\perp}$, where the right action of $\operatorname{Per}(\Lambda)^{\perp}$ is given by $(z, I) \cdot w:=\left(z w, \alpha_{w^{-1}}(I)\right)$ [39, Lemma 2.8]. In particular, since $C^{*}(H \Lambda) \cong \operatorname{Ind}(\alpha)$, we obtain $\operatorname{Prim}\left(C^{*}(H \Lambda)\right) \cong \operatorname{Prim}(\operatorname{Ind}(\alpha)) \cong\left(\mathbb{T}^{k} \times \operatorname{Prim}\left(C^{*}(\Gamma)\right)\right) / \operatorname{Per}(\Lambda)^{\perp}$.

Lemma 3.5. Let $\Lambda$ and $\Gamma$ be as in Lemma 3.3. The formula

$$
\varphi\left((z, I) \cdot \operatorname{Per}(\Lambda)^{\perp}\right)=z \cdot \operatorname{Per}(\Lambda)^{\perp},
$$

determines a continuous open surjection $\varphi:\left(\mathbb{T}^{k} \times \operatorname{Prim}\left(C^{*}(\Gamma)\right)\right) / \operatorname{Per}(\Lambda)^{\perp} \rightarrow \mathbb{T}^{k} / \operatorname{Per}(\Lambda)^{\perp}$.

Proof. For completeness let us mention that $\operatorname{Prim}\left(C^{*}(\Gamma)\right)$ is non-empty (for example, the zero ideal in $C^{*}(\Gamma)$ is primitive) so $\varphi$ makes sense. If $\left(x_{1}, I_{1}\right) \cdot \operatorname{Per}(\Lambda)^{\perp}=\left(x_{2}, I_{2}\right) \cdot \operatorname{Per}(\Lambda)^{\perp}$ for $\left(x_{i}, I_{i}\right) \in \mathbb{T}^{k} \times \operatorname{Prim}\left(C^{*}(\Gamma)\right)$, then $x_{1} w=x_{2}$ for some $w \in \operatorname{Per}(\Lambda)^{\perp}$. So the map $\varphi$ is well defined.

Let $\psi: \mathbb{T}^{k} \times \operatorname{Prim}\left(C^{*}(\Gamma)\right) \rightarrow \mathbb{T}^{k}$ be the projection onto the first coordinate and write $p$ for both the quotient map $\mathbb{T}^{k} \rightarrow \mathbb{T}^{k} / \operatorname{Per}(\Lambda)^{\perp}$ and the orbit map $\mathbb{T}^{k} \times \operatorname{Prim}\left(C^{*}(\Gamma)\right) \rightarrow$ $\left(\mathbb{T}^{k} \times \operatorname{Prim}\left(C^{*}(\Gamma)\right)\right) / \operatorname{Per}(\Lambda)^{\perp}$ (cf. [39, Definition 3.21]). Then the diagram

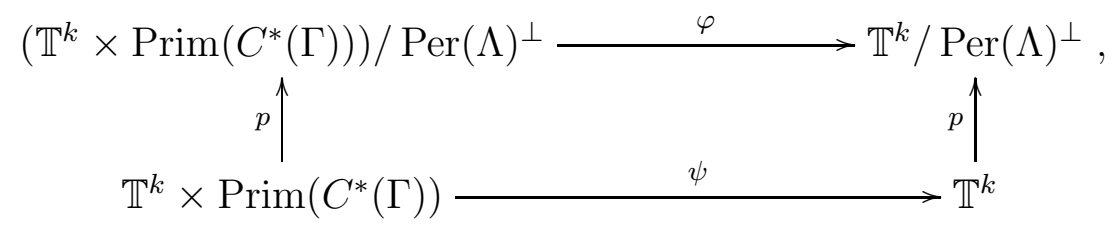

commutes. The topologies on the orbit spaces

$$
\left(\mathbb{T}^{k} \times \operatorname{Prim}\left(C^{*}(\Gamma)\right)\right) / \operatorname{Per}(\Lambda)^{\perp} \quad \text { and } \quad \mathbb{T}^{k} / \operatorname{Per}(\Lambda)^{\perp}
$$

are the weakest topologies making the maps $p$ continuous. So both maps $p$ are continuous and open (see [39, Lemma 3.25]). Now $\varphi$ is continuous and open because $\psi$ is. The map $\varphi$ is surjective because $\operatorname{Prim}\left(C^{*}(\Gamma)\right)$ is non-empty. 
We will need an elementary lemma from topology. This is standard, but we give a complete statement and proof for convenience.

Lemma 3.6. Let $X, Y$ be topological spaces and let $\varphi: X \rightarrow Y$ be a continuous open surjective map. If $X$ admits a basis for its topology consisting of compact and open sets, then so does $Y$.

Proof. Let $\mathbb{B}$ be a basis for the topology on $X$ consisting of compact and open sets. Set $\mathbb{F}:=\{\varphi(B): B \in \mathbb{B}\}$. Since $\varphi$ is continuous and open each element of $\mathbb{F}$ is compact and open. Since $\varphi$ is surjective $Y=\varphi\left(\bigcup_{B \in \mathbb{B}} B\right)=\bigcup_{F \in \mathbb{F}} F$, so $\mathbb{F}$ is an open cover of $Y$. Suppose $y \in F_{1} \cap F_{2}$ for $F_{1}, F_{2} \in \mathbb{F}$. Take $x \in X$ such that $\varphi(x)=y$. Since $x \in \varphi^{-1}(y) \subseteq$ $\varphi^{-1}\left(F_{i}\right)$ and $F_{i}$ is open, there exists $B \in \mathbb{B}$ such that $x \in B \subseteq \varphi^{-1}\left(F_{1}\right) \cap \varphi^{-1}\left(F_{2}\right)$. With $F:=\varphi(B) \in \mathbb{F}$ we get $y=\varphi(x) \in \varphi(B)=F$ and $F=\varphi(B) \subseteq \varphi\left(\varphi^{-1}\left(F_{i}\right)\right)=F_{i}$. So $\mathbb{F}$ is a basis for a topology on $Y$.

To see that this topology coincides with the given topology on $Y$, observe that each set in $\mathbb{F}$ is open, and for any open set $V \subseteq Y$, the preimage $\varphi^{-1}(V)$ is open, hence a union of elements $B_{i} \in \mathbb{B}$. Now each $\varphi\left(B_{i}\right) \in \mathbb{F}$ and $\left.V=\varphi\left(\varphi^{-1}(V)\right)=\varphi\left(\bigcup_{i} B_{i}\right)\right)=\bigcup_{i} \varphi\left(B_{i}\right)$.

We write $\mathcal{M}(A)$ for the multiplier algebra of a $C^{*}$-algebra $A$.

Lemma 3.7. Let $\Lambda$ be a row-finite $k$-graph with no sources. Suppose that $C^{*}(\Lambda)$ has topological dimension zero. Suppose that $H \subseteq \Lambda^{0}$ is hereditary. Let I be the ideal of $C^{*}(\Lambda)$ generated by $\left\{p_{v}: v \in H\right\}$, and let $P_{H}:=\sum_{v \in H} p_{v} \in \mathcal{M}(I)$. There is an isomorphism $C^{*}(H \Lambda) \cong P_{H} I P_{H}$ carrying the generator $s_{\lambda}$ of $C^{*}(H \Lambda)$ to the corresponding generator $s_{\lambda}$ of $C^{*}(\Lambda)$ for every $\lambda \in H \Lambda$, and $P_{H} I P_{H}$ is a full corner of $I$. Furthermore, $C^{*}(H \Lambda)$ has topological dimension zero. If $H$ is also saturated then $C^{*}(\Lambda \backslash \Lambda H)$ is isomorphic to $C^{*}(\Lambda) / I$ and has topological dimension zero.

Proof. Recall from [8, Proposition 3.2.1] and [5, Proposition 2.8] that topological dimension zero passes to ideals and quotients. It also passes to full corners (since Morita equivalent $C^{*}$-algebras have homeomorphic primitive ideal spaces, see [7, p. 156]).

Let $H$ be any hereditary subset of $\Lambda^{0}$. By [32, Theorem 5.2], $C^{*}(H \Lambda)$ is isomorphic to $P_{H} I P_{H}$. This is a full corner because $I$ is generated by $P_{H}$. Since topological dimension zero passes to ideals and full corners, we deduce that $C^{*}(H \Lambda)$ has topological dimension zero. If $H$ is saturated as well, then $C^{*}(\Lambda \backslash \Lambda H)$ is isomorphic to $C^{*}(\Lambda) / I$, and hence it, too, has topological dimension zero.

Lemma 3.8. Let $\Lambda$ be a row-finite $k$-graph with no sources. Suppose that $\Lambda$ is not strongly aperiodic. Then there exists a (possibly empty) hereditary and saturated set $H \subseteq \Lambda^{0}$ such that the vertex set of $\Lambda \backslash \Lambda H$ is a maximal tail and $\operatorname{Per}(\Lambda \backslash \Lambda H) \neq\{0\}$.

Proof. Choose a (possibly empty) hereditary saturated set $H^{\prime} \subseteq \Lambda^{0}$ such that $\Lambda \backslash \Lambda H^{\prime}$ is not aperiodic. By [36, Lemma 3.2(iii)] there exist a vertex $v \in \Lambda \backslash \Lambda H^{\prime}$ and $n \neq m \in \mathbb{N}^{k}$ such that $\sigma^{m}(y)=\sigma^{n}(y)$ for every infinite path $y \in v\left(\Lambda \backslash \Lambda H^{\prime}\right)^{\infty}$. Fix $x \in v\left(\Lambda \backslash \Lambda H^{\prime}\right)^{\infty}$, define the shift-tail equivalence class

$$
[x]:=\left\{\lambda \sigma^{p}(x): p \in \mathbb{N}^{k}, \lambda \in\left(\Lambda \backslash \Lambda H^{\prime}\right) x(p)\right\},
$$

and let $T:=r([x]) \subseteq \Lambda^{0}$ (possibly all of $\Lambda^{0}$ ) and $H:=\Lambda^{0} \backslash T$.

We prove that $T \subseteq \Lambda^{0}$ is a maximal tail. First we consider property (a). If $v, w \in r([x])$, say $v=r\left(\alpha \sigma^{m}(x)\right), w=r\left(\beta \sigma^{n}(x)\right)$, then $u=x(m \vee n) \in T$ satisfies $\alpha x(m, m \vee n) \in v \Lambda u$, and $\beta x(n, m \vee n) \in w \Lambda u$. For property (b), fix $v \in r([x])$, say $v=r\left(\alpha \sigma^{m}(x)\right)$, and fix 
$i \leq k$. By replacing $\alpha$ with $\alpha x\left(m, m+e_{i}\right)$ and $m$ with $m+e_{i}$, we may assume that $d(\alpha) \geq e_{i}$, say $\alpha=f \alpha^{\prime}$ with $f \in \Lambda^{e_{i}}$. Then $f \in v \Lambda^{e_{i}}$, and $s(f)=r\left(\alpha^{\prime} \sigma^{m}(x)\right) \in r([x])$. Finally for property (c), fix $w \in r([x])$ and $v \in \Lambda^{0}$ with $v \Lambda w \neq \emptyset$, say $\alpha \in v \Lambda w$. Write $w=r\left(\beta \sigma^{m}(x)\right)$. Then $v=r\left((\alpha \beta) \sigma^{m}(x)\right) \in r([x])$.

Now $H \subseteq \Lambda^{0}$ is hereditary and saturated because it is the complement of a maximal tail (see the proof of [16, Theorem 3.12]). Since $T(\Lambda \backslash \Lambda H) T=\Lambda \backslash \Lambda H$, the set $T=(\Lambda \backslash \Lambda H)^{0}$ is a maximal tail in the $k$-graph $\Lambda \backslash \Lambda H$.

Since $\operatorname{Per}(\Lambda \backslash \Lambda H)=\left\{a-b\right.$ : there exists $w \in(\Lambda \backslash \Lambda H)^{0}$ such that $\sigma^{a}(y)=\sigma^{b}(y)$ for all $\left.y \in w(\Lambda \backslash \Lambda H)^{\infty}\right\}$ and since $(\Lambda \backslash \Lambda H)^{0}=r([x]) \subseteq\left\{r(\lambda): \lambda \in \Lambda \backslash \Lambda H^{\prime}\right\}=\left(\Lambda \backslash \Lambda H^{\prime}\right)^{0}$ we conclude that $n-m \in \operatorname{Per}(\Lambda \backslash \Lambda H) \neq\{0\}$.

Proof of Theorem [3.2. As mentioned before, (ii) $\Rightarrow$ (i) follows from [16, Theorem 4.2]. For (i) $\Rightarrow$ (ii), let $\Lambda^{\prime}$ be a row-finite $k$-graph with no sources such that $C^{*}\left(\Lambda^{\prime}\right)$ has topological dimension zero. We suppose that $\Lambda^{\prime}$ is not strongly aperiodic, and derive a contradiction. By Lemma 3.8 there is a hereditary and saturated set $H^{\prime} \subseteq\left(\Lambda^{\prime}\right)^{0}$ such that the vertex set $\Lambda^{0}$ of $\Lambda:=\Lambda^{\prime} \backslash \Lambda^{\prime} H^{\prime}$ is a maximal tail and such that $\operatorname{Per}(\Lambda)$ is nontrivial. Define $H:=H_{\text {Per }}$ and $\Gamma:=(H \Lambda) / \sim$. By Lemma 3.4. $\operatorname{Prim}\left(C^{*}(H \Lambda)\right) \cong\left(\mathbb{T}^{k} \times \operatorname{Prim}\left(C^{*}(\Gamma)\right)\right) / \operatorname{Per}(\Lambda)^{\perp}$. By Lemma 3.5, there is a continuous open surjective map

$$
\varphi:\left(\mathbb{T}^{k} \times \operatorname{Prim}\left(C^{*}(\Gamma)\right)\right) / \operatorname{Per}(\Lambda)^{\perp} \rightarrow \mathbb{T}^{k} / \operatorname{Per}(\Lambda)^{\perp} .
$$

Lemma 3.7 implies that $C^{*}(H \Lambda)$ has topological dimension zero, and it follows that $\left(\mathbb{T}^{k} \times\right.$ $\left.\operatorname{Prim}\left(C^{*}(\Gamma)\right)\right) / \operatorname{Per}(\Lambda)^{\perp}$ has a basis of compact open sets. Using Lemma 3.6 we conclude that $\mathbb{T}^{k} / \operatorname{Per}(\Lambda)^{\perp}$ has a basis of compact open sets. But $\mathbb{T}^{k} / \operatorname{Per}(\Lambda)^{\perp}$ is the Pontryagin dual of a free abelian group, and hence homeomorphic to $\mathbb{T}^{l}$ where $l$ is the rank of $\operatorname{Per}(\Lambda)$. So $\mathbb{T}^{k} / \operatorname{Per}(\Lambda)^{\perp}$ is connected and not a singleton, a contradiction.

Recall that a topological groupoid $\mathcal{G}$ is topologically principal if the set

$$
\left\{u \in \mathcal{G}^{(0)}: \mathcal{G}_{u}^{u}=\{u\}\right\}
$$

of units with trivial isotropy is dense in $\mathcal{G}^{(0)}$ [34, Definition 1.2], and essentially principal if for every closed invariant $X \subseteq \mathcal{G}^{(0)}$, the set $\left\{u \in X: \mathcal{G}_{u}^{u}=\{u\}\right\}$ is dense in $X$ [33, Definition 4.3]. For each $k$-graph $\Lambda$ with no sources we let $\mathcal{G}_{\Lambda}$ denote the infinite-path groupoid of $\Lambda$ introduced in [21, Definition 2.7].

Corollary 3.9. Let $\Lambda$ be a row-finite k-graph with no sources. Then the following are equivalent:

(i) The k-graph $\Lambda$ is strongly aperiodic.

(ii) For every hereditary saturated subset $H \subsetneq \Lambda^{0}$, the groupoid $\mathcal{G}_{\Lambda \backslash \Lambda H}$ is topologically principal.

(iii) The groupoid $\mathcal{G}_{\Lambda}$ is essentially principal.

(iv) The $C^{*}$-algebra $C^{*}(\Lambda)$ has topological dimension zero.

(v) Every ideal of $C^{*}(\Lambda)$ is gauge invariant.

(vi) The map $I \mapsto\left\{v \in \Lambda^{0}: s_{v} \in I\right\}$ is a bijection between ideals of $C^{*}(\Lambda)$ and hereditary saturated subsets $H \subseteq \Lambda^{0}$.

Proof. See [21, Proposition 4.5] for (i) $\Leftrightarrow$ (ii). For (ii) $\Leftrightarrow$ (iii), one checks that the nonempty closed invariant subsets of $\mathcal{G}_{\Lambda}^{(0)}$ are precisely the sets $(\Lambda \backslash \Lambda H)^{\infty} \subseteq \Lambda^{\infty}$ associated to hereditary saturated sets $H \subsetneq \Lambda^{0}$. This follows from the argument of [22, Proposition 6.5] mutatis mutandis. 
Theorem 3.2 gives $(\mathrm{i}) \Leftrightarrow(\mathrm{iv})$. For $(\mathrm{i}) \Leftrightarrow(\mathrm{v})$ see [36, Proposition 3.6] and see [37, Theorem 5.5] for $(\mathrm{v}) \Rightarrow(\mathrm{vi})$. For $(\mathrm{vi}) \Rightarrow(\mathrm{v})$ observe that the $s_{v}$ are fixed under the gauge action, so any ideal generated by vertex projections is gauge invariant.

\section{REAL RANK ZERO}

In this section we study when $k$-graph $C^{*}$-algebras have real-rank zero. We can give a complete answer for 2-graphs $\Lambda$ such that $C^{*}(\Lambda)$ is strongly purely infinite, and we are able to say some things in greater generality. We start with a necessary condition that follows easily from work of Brown-Pedersen (it could also be deduced easily from work of Pasnicu [29]) and requires no additional hypotheses. It also connects the question of real-rank zero to our work in the preceding section.

Lemma 4.1. Let $\Lambda$ be a row-finite $k$-graph with no sources. If the $C^{*}$-algebra $C^{*}(\Lambda)$ has real-rank zero then $\Lambda$ is strongly aperiodic.

Proof. Suppose that $C^{*}(\Lambda)$ has real-rank zero. Then it has generalised real-rank zero in the sense of [5, 2.1(iv)], and so Proposition 2.7 of [5] implies that it also has topological dimension zero. Hence (i) $\Longrightarrow$ (ii) of Theorem 3.2 implies that $\Lambda$ is strongly aperiodic.

Our objective in the rest of the section is to strengthen this necessary condition and obtain a necessary and sufficient condition for real-rank zero when $C^{*}(\Lambda)$ is purely infinite and $k=2$.

For an $R$-module $M$ and an integer $a \geq 2$ let $\bigwedge^{a} M$ denote the ath exterior power of $M$, i.e. the $R$-module $M^{\otimes a} / J_{a}$ where $J_{a}$ is the submodule of $M^{\otimes a}$ spanned by elements of the form $m_{1} \otimes m_{2} \otimes \cdots \otimes m_{a}$ such that $m_{i}=m_{j}$ for some $i \neq j$. For any $m_{1}, m_{2}, \ldots, m_{a} \in M$, the coset of $m_{1} \otimes m_{2} \otimes \cdots \otimes m_{a}$ in $\wedge^{a} M$ is denoted $m_{1} \wedge m_{2} \wedge \cdots \wedge m_{a}$ and we call it an elementary wedge product. If $\left\{\epsilon_{1}, \epsilon_{2}, \ldots, \epsilon_{N}\right\}$ constitutes a basis for $M$ (with $a \leq N$ ) then $\left\{\epsilon_{i_{1}} \wedge \epsilon_{i_{2}} \wedge \cdots \wedge \epsilon_{i_{a}}: 1 \leq i_{1}<\cdots<i_{a} \leq N\right\}$ is a basis for $\wedge^{a} M$. We regard $R$ as an $R$-module and set $\bigwedge^{0} M=R$ and $\bigwedge^{1} M=M$.

Let $\Lambda$ be a row finite $k$-graph with no sources. Following [23] we introduce the chain complex $D_{*}^{\Lambda}$. Let $\mathbb{Z} \Lambda^{0}$ denote the set of finitely supported functions from $\Lambda^{0}$ to $\mathbb{Z}$, regarded as an abelian group under pointwise addition. For $v \in \Lambda^{0}$ define $\delta_{v} \in \mathbb{Z} \Lambda^{0}$ by $\delta_{v}(u)=\delta_{u, v}$ (the Kronecker delta). For each $i=1, \ldots, k$ let $M_{i}$ (or $M_{i, \Lambda}$ for emphasis) be the vertex connectivity matrix given by $M_{i}(u, v)=\left|u \Lambda^{e_{i}} v\right|$. Regard $M_{i}$ as the group endomorphism of $\mathbb{Z} \Lambda^{0}$ given by $\left(M_{i} f\right)(u)=\sum_{v \in \Lambda^{0}} M_{i}(u, v) f(v)$. Let $D_{*}^{\Lambda}$ be the chain complex such that

$$
D_{a}^{\Lambda}= \begin{cases}\bigwedge^{a} \mathbb{Z}^{k} \otimes \mathbb{Z} \Lambda^{0} & \text { if } 0 \leq a \leq k \\ 0 & \text { if } a>k\end{cases}
$$

with differentials $\partial_{a}: D_{a}^{\Lambda} \rightarrow D_{a-1}^{\Lambda}$ (or $\partial_{a}^{\Lambda}$ for emphasis) defined for $1 \leq a \leq k$ by

$$
\partial_{a}\left(\epsilon_{i_{1}} \wedge \cdots \wedge \epsilon_{i_{a}} \otimes \delta_{v}\right)=\sum_{j=1}^{a}(-1)^{j+1} \epsilon_{i_{1}} \wedge \cdots \wedge \widehat{\epsilon}_{i_{j}} \wedge \cdots \wedge \epsilon_{i_{a}} \otimes\left(1-M_{j}^{t}\right) \delta_{v},
$$

where the symbol " $\uparrow$ " denotes deletion of an element, $v \in \Lambda^{0},\left\{\epsilon_{1}, \ldots, \epsilon_{k}\right\}$ is the canonical basis for $\mathbb{Z}^{k}$ and $M_{j}^{t}$ is the transpose of $M_{j}$.

Example 4.2. Let $\Lambda$ be a row finite $k$-graph with no sources, $H \subsetneq \Lambda^{0}$ a hereditary saturated subset of $\Lambda^{0}$ and $\Gamma=H \Lambda$. Recall that a morphism of chain complexes $f: C_{*} \rightarrow D_{*}$ is 
given by a family of morphisms $f_{n}: C_{n} \rightarrow D_{n}$ that commute with the differentials. In this example we construct a morphism of chain complexes $j^{H}: D_{*}^{\Gamma} \rightarrow D_{*}^{\Lambda}$.

For each $f \in \mathbb{Z} \Gamma^{0}$ let $i^{H}(f)$ be the extension of $f$ to $\Lambda^{0}$ by zero. Since $H$ is hereditary, for $u \in H$ and $v \notin H$ we have $M_{j, \Lambda}(u, v)=0$. It follows that for each $v \in \Lambda^{0}$

$$
\begin{aligned}
\left(M_{j, \Lambda}^{t}\left(i^{H}(f)\right)\right)(v) & =\sum_{u \in \Lambda^{0}} M_{j, \Lambda}(u, v)\left(i^{H}(f)(u)\right)=\sum_{u \in \Gamma^{0}} M_{j, \Lambda}(u, v)(f(u)) \\
& =1_{\Gamma^{0}}(v) \sum_{u \in \Gamma^{0}} M_{j, \Lambda}(u, v)(f(u))=\left(i^{H}\left(M_{j, \Gamma}^{t} f\right)\right)(v) .
\end{aligned}
$$

We conclude that $i^{H}\left(\left(1-M_{j, \Gamma}^{t}\right) f\right)=\left(1-M_{j, \Lambda}^{t}\right)\left(i^{H}(f)\right)$ for each $f \in \mathbb{Z} \Gamma^{0}$. For $0 \leq a \leq k$ let $j_{a}^{H}: D_{a}^{\Gamma} \rightarrow D_{a}^{\Lambda}$ be the morphism given by $j_{a}^{H}(d \otimes f)=d \otimes i^{H}(f)$. The above calculation produces the commuting diagram

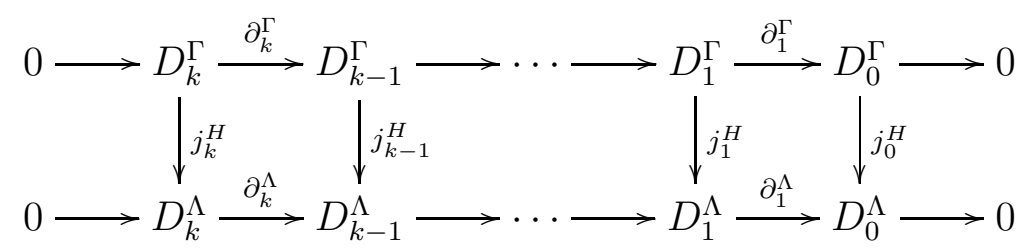

and hence yields a morphism of chain complexes $j^{H}: D_{*}^{\Gamma} \rightarrow D_{*}^{\Lambda}$.

Any morphism of chain complexes induces a morphism of the homology groups of the chain complexes. In particular Example 4.2 yields the morphism $H_{*}\left(j^{H}\right): H_{*}\left(D_{*}^{\Gamma}\right) \rightarrow$ $H_{*}\left(D_{*}^{\Lambda}\right)$. We can now state our main result.

Theorem 4.3. Let $\Lambda$ be a row-finite 2-graph with no sources.

(1) Suppose $C^{*}(\Lambda)$ is purely infinite. Then the following are equivalent:

(i) $C^{*}(\Lambda)$ has real rank zero

(ii) $\Lambda$ is strongly aperiodic and $H_{1}\left(j^{H}\right)$ is injective for every saturated hereditary subset $H$ of $\Lambda^{0}$.

(2) The implication (i) $\Rightarrow$ (ii) holds even if $C^{*}(\Lambda)$ is not purely infinite.

We will prove Theorem 4.3 at the end of the section, after establishing some preliminary results. First though, we present a reformulation of the condition that $H_{1}\left(j^{H}\right)$ is injective in terms of the connectivity matrices of the 2-graph $\Lambda$. This result will prove useful when applying Theorem 4.3 in practice. To state it, we need a little bit more notation. Suppose that $\Lambda$ is a 2-graph and $H \subseteq \Lambda^{0}$ is a hereditary set. It follows that, for each $i$, $M_{i, \Lambda}(u, v)=0$ whenever $u \in H$ and $v \notin H$. This means that each $M_{i, \Lambda}^{t}$ has a block-uppertriangular decomposition with respect to the decomposition $\Lambda^{0}=H \sqcup\left(\Lambda^{0} \backslash H\right)$. We will use the following notation for this decomposition:

$$
M_{i, \Lambda}^{t}=\left(\begin{array}{cc}
M_{i, H}^{t} & M_{i, H, \Lambda^{0} \backslash H}^{t} \\
0 & M_{i, \Lambda^{0} \backslash H}^{t}
\end{array}\right) .
$$

Proposition 4.4. Let $\Lambda$ be a row-finite 2-graph with no sources, and let $H \subseteq \Lambda^{0}$ be a saturated hereditary set. With $H_{1}\left(j^{H}\right), \partial_{1}, \partial_{2}$ and $\Gamma$ as in Example 4.2, the following are equivalent:

(i) The morphism $H_{1}\left(j^{H}\right)$ is injective.

(ii) We have im $\partial_{2}^{\Lambda} \cap j_{1}^{H}\left(\operatorname{ker} \partial_{1}^{\Gamma}\right) \subseteq j_{1}^{H}\left(\operatorname{im} \partial_{2}^{\Gamma}\right)$. 
(iii) We have

$$
\left(\begin{array}{l}
M_{1, H, \Lambda^{0} \backslash H}^{t} \\
M_{2, H, \Lambda^{0} \backslash H}^{t}
\end{array}\right)\left(\operatorname{ker}\left(M_{1, \Lambda^{0} \backslash H}^{t}-1\right) \cap \operatorname{ker}\left(M_{2, \Lambda^{0} \backslash H}^{t}-1\right)\right) \subseteq\left(\begin{array}{l}
M_{1, H}^{t}-1 \\
M_{2, H}^{t}-1
\end{array}\right) \mathbb{Z} H .
$$

Proof. Before proving (i) $\Leftrightarrow($ ii), we do some initial work to reformulate condition (i). Since $j_{1}^{H} \circ \partial_{2}^{\Gamma}=\partial_{2}^{\Lambda} \circ j_{2}^{H}$, we have $j_{1}^{H}\left(\operatorname{im} \partial_{2}^{\Lambda}\right) \subseteq \operatorname{im} \partial_{2}^{\Lambda}$. Hence there is a well-defined homomorphism $H_{1}\left(j^{H}\right): \operatorname{ker} \partial_{1}^{\Gamma} / \operatorname{im} \partial_{2}^{\Gamma} \rightarrow \operatorname{ker} \partial_{1}^{\Lambda} / \operatorname{im} \partial_{2}^{\Lambda}$ such that

$$
H_{1}\left(j^{H}\right)\left(a+\operatorname{im} \partial_{2}^{\Gamma}\right)=j_{1}^{H}(a)+\operatorname{im} \partial_{2}^{\Lambda} \quad \text { for all } a \in \operatorname{ker} \partial_{1}^{\Gamma} .
$$

By linearity, it follows that $H_{1}\left(j^{H}\right)$ is injective if and only if whenever $a \in \operatorname{ker} \partial_{1}^{\Gamma}$ satisfies $j_{1}^{H}(a) \in \operatorname{im} \partial_{2}^{\Lambda}$, we have $a \in \operatorname{im} \partial_{2}^{\Gamma}$.

Now, to prove $(\mathrm{i}) \Rightarrow(\mathrm{ii})$, suppose that $H_{1}\left(j^{H}\right)$ is injective, and fix $a \in \operatorname{ker} \partial_{1}^{\Gamma}$ such that $j_{1}^{H}(a) \in \operatorname{im} \partial_{2}^{\Lambda}$. Then the preceding paragraph shows that $a \in \operatorname{im} \partial_{2}^{\Gamma}$, giving $j_{1}^{H}(a) \in$ $j_{1}^{H}\left(\operatorname{im} \partial_{2}^{\Gamma}\right)$.

To verify (ii) $\Rightarrow(\mathrm{i})$, we suppose that (ii) holds, and fix $a \in \operatorname{ker} \partial_{1}^{\Gamma}$ with $j_{1}^{H}(a) \in \operatorname{im} \partial_{2}^{\Lambda}$. By the first paragraph of the proof, it suffices to show that $a \in \operatorname{im} \partial_{2}^{\Gamma}$. We have $j_{1}^{H}(a) \in$ $\operatorname{im} \partial_{2}^{\Lambda} \cap j_{1}^{H}\left(\operatorname{ker} \partial_{1}^{\Gamma}\right)$. By (ii), $j_{1}^{H}(a) \in j_{1}^{H}\left(\operatorname{im} \partial_{2}^{\Gamma}\right)$, so $j_{1}^{H}(a)=j_{1}^{H}(c)$ for some $c \in \operatorname{im} \partial_{2}^{\Gamma}$. Since $j_{1}^{H}(d \otimes f)=d \otimes i^{H}(f)$, where $i^{H}(f)$ is the extension of $f \in \mathbb{Z} \Gamma^{0}$ to $\Lambda^{0}$ by zero, $j_{1}^{H}$ is injective, so $a=c \in \operatorname{im} \partial_{2}^{\Gamma}$.

We now prove that (ii) $\Leftrightarrow\left(\right.$ iii). By [10, 23], the complex $D_{a}^{\Lambda}=\bigwedge^{a} \mathbb{Z}^{k} \otimes \mathbb{Z} \Lambda^{0}$ may be written as follows:

$$
0 \longrightarrow \mathbb{Z} \Lambda^{0} \stackrel{\partial_{2}^{\Lambda}}{\longrightarrow} \mathbb{Z} \Lambda^{0} \oplus \mathbb{Z} \Lambda^{0} \stackrel{\partial_{1}^{\Lambda}}{\longrightarrow} \mathbb{Z} \Lambda^{0} \longrightarrow 0
$$

where $\partial_{1}^{\Lambda}=\left(1-M_{1, \Lambda}^{t}, 1-M_{2, \Lambda}^{t}\right)$ and $\partial_{2}^{\Lambda}=\left(\begin{array}{c}M_{2, \Lambda}^{t}-1 \\ 1-M_{1, \Lambda}^{t}\end{array}\right)$. The same applies to the 2-graph $\Gamma=H \Lambda$. To ease notation in the calculations that follow, we write $T:=\Lambda^{0} \backslash H$, and we write $A:=M_{1, \Lambda}^{t}$ and $B:=M_{2, \Lambda}^{t}$, so that

$$
1-M_{1, \Lambda}^{t}=\left(\begin{array}{cc}
1-A_{H} & -A_{H, T} \\
0 & 1-A_{T}
\end{array}\right) \quad \text { and } \quad 1-M_{2, \Lambda}^{t}=\left(\begin{array}{cc}
1-B_{H} & -B_{H, T} \\
0 & 1-B_{T}
\end{array}\right) .
$$

Define

$$
K_{1}:=\operatorname{im} \partial_{2}^{\Lambda}, \quad K_{2}:=j_{1}^{H}\left(\operatorname{ker} \partial_{1}^{\Gamma}\right), \quad \text { and } \quad L_{1}:=j_{1}^{H}\left(\operatorname{im} \partial_{2}^{\Gamma}\right),
$$

so that (ii) is satisfied if and only if $K_{1} \cap K_{2} \subseteq L_{1}$. We have

$$
\begin{aligned}
K_{1} & =\left\{\left(\left(\begin{array}{cc}
B_{H}-1 & B_{H, T} \\
0 & B_{T}-1
\end{array}\right)\left(\begin{array}{l}
a \\
b
\end{array}\right),\left(\begin{array}{cc}
1-A_{H} & -A_{H, T} \\
0 & 1-A_{T}
\end{array}\right)\left(\begin{array}{l}
a \\
b
\end{array}\right)\right):\left(\begin{array}{l}
a \\
b
\end{array}\right) \in \mathbb{Z}^{0}\right\}, \\
K_{2} & =j_{1}^{H}\left(\left\{(u, v): u, v \in \mathbb{Z} H \text { and } A_{H} u+B_{H} v=u+v\right\}\right) \\
& =\left\{\left(\left(\begin{array}{c}
u \\
0
\end{array}\right),\left(\begin{array}{c}
v \\
0
\end{array}\right)\right): u, v \in \mathbb{Z} H \text { and } A_{H} u+B_{H} v=u+v\right\}, \text { and } \\
L_{1} & =j_{1}^{H}\left(\left\{\left(\left(B_{H}-1\right) a,\left(1-A_{H}\right) a\right): a \in \mathbb{Z} H\right\}\right) \\
& =\left\{\left(\left(\begin{array}{c}
\left(B_{H}-1\right) a \\
0
\end{array}\right),\left(\begin{array}{c}
\left(1-A_{H}\right) a \\
0
\end{array}\right)\right): a \in \mathbb{Z} H\right\} .
\end{aligned}
$$

Suppose that $\left(\left(\begin{array}{l}u \\ 0\end{array}\right),\left(\begin{array}{l}v \\ 0\end{array}\right)\right) \in K_{1} \cap K_{2}$. The description of $K_{1}$ shows that $\left(\left(\begin{array}{l}u \\ 0\end{array}\right),\left(\begin{array}{l}v \\ 0\end{array}\right)\right)$ has the form $\partial_{2}^{\Lambda}\left(\begin{array}{c}a \\ b\end{array}\right)$ where $b \in \operatorname{ker}\left(1-B_{T}\right) \cap \operatorname{ker}\left(1-A_{T}\right)$. Conversely, if $b \in \operatorname{ker}\left(1-B_{T}\right) \cap \operatorname{ker}\left(1-A_{T}\right)$ 
and $a \in \mathbb{Z} H$, then we have

$$
\partial_{2}^{\Lambda}\left(\begin{array}{l}
a \\
b
\end{array}\right)=\left(\left(\begin{array}{c}
\left(B_{H}-1\right) a+B_{H, T} b \\
0
\end{array}\right),\left(\begin{array}{c}
\left(1-A_{H}\right) a-A_{H, T} b \\
0
\end{array}\right)\right) .
$$

For $a \in \mathbb{Z} \Lambda^{0}$, we write $a_{H}$ for the component $\left(a_{v}\right)_{v \in H}$ of $a$ belonging to $\mathbb{Z} H$. Using that $M_{1, \Lambda}^{t}$ and $M_{2, \Lambda}^{t}$ commute, we have

$$
\begin{aligned}
A_{H}\left(\left(B_{H}-1\right) a+B_{H, T} b\right)+ & B_{H}\left(\left(1-A_{H}\right) a-A_{H, T} b\right) \\
& =\left(M_{1, \Lambda}^{t}\left(M_{2, \Lambda}^{t}-1\right)\left(\begin{array}{c}
a \\
b
\end{array}\right)\right)_{H}+\left(M_{2, \Lambda}^{t}\left(1-M_{1, \Lambda}^{t}\right)\left(\begin{array}{c}
a \\
b
\end{array}\right)\right)_{H} \\
& =\left(\left(M_{2, \Lambda}^{t}-1\right)\left(\begin{array}{c}
a \\
b
\end{array}\right)\right)_{H}+\left(\left(1-M_{1, \Lambda}^{t}\right)\left(\begin{array}{c}
a \\
b
\end{array}\right)\right)_{H} \\
& =\left(\left(B_{H}-1\right) a+B_{H, T} b\right)+\left(\left(1-A_{H}\right) a-A_{H, T} b\right) .
\end{aligned}
$$

Thus $\partial_{2}^{\Lambda}\left(\begin{array}{l}a \\ b\end{array}\right)$ belongs to $K_{1} \cap K_{2}$. That is, we have showed that

$$
K_{1} \cap K_{2}=\left\{\partial_{2}^{\Lambda}\left(\begin{array}{l}
a \\
b
\end{array}\right): a \in \mathbb{Z} H \text { and } b \in \operatorname{ker}\left(1-B_{T}\right) \cap \operatorname{ker}\left(1-A_{T}\right)\right\} .
$$

Let

$$
L_{2}:=\left\{\left(\left(\begin{array}{c}
B_{H, T} b \\
0
\end{array}\right),\left(\begin{array}{c}
-A_{H, T^{b}} \\
0
\end{array}\right)\right): b \in \mathbb{Z} T \text { and } b \in \operatorname{ker}\left(1-B_{T}\right) \cap \operatorname{ker}\left(1-A_{T}\right)\right\} .
$$

We have shown that $K_{1} \cap K_{2}=L_{1}+L_{2}$, and hence (ii) is satisfied if and only if $L_{2} \subseteq L_{1}$. As the pairs of vectors in $L_{1}$ and $L_{2}$ have zeros at the second row, it follows that (ii) holds if and only if

$$
\begin{aligned}
\left\{\left(B_{H, T} b,-A_{H, T} b\right): b \in \mathbb{Z} T\right. & \text { and } \left.b \in \operatorname{ker}\left(1-B_{T}\right) \cap \operatorname{ker}\left(1-A_{T}\right)\right\} \\
& \subseteq\left\{\left(\left(B_{H}-1\right) a,\left(1-A_{H}\right) a\right): a \in \mathbb{Z} H\right\} .
\end{aligned}
$$

Multiplying by -1 in the second coordinate and recalling the definitions of the matrices $A$ and $B$ gives (ii) $\Leftrightarrow($ iii).

We now embark on the preliminary results needed for the proof of Theorem 4.3. Let $\Lambda$ be a row finite $k$-graph with no sources. As usual, given an action $\alpha$ of a locally compact group $G$ on a $C^{*}$-algebra $A$, we write $i_{G}: C^{*}(G) \rightarrow \mathcal{M}\left(A \times_{\alpha} G\right)$ and $i_{A}: A \rightarrow \mathcal{M}\left(A \times_{\alpha} G\right)$ for the canonical inclusions into the multiplier algebra of the crossed product.

We will abbreviate the crossed product $C^{*}(\Lambda) \times_{\gamma} \mathbb{T}^{k}$ of $C^{*}(\Lambda)$ by its gauge action by $B^{\Lambda}$. For each $n \in \mathbb{Z}^{k}$ and $f \in C_{c}\left(\mathbb{T}^{k}, C^{*}(\Lambda)\right)$ define $\alpha_{n}^{\Lambda}(f)(z):=z^{-n} f(z)$. Then $\alpha_{n}^{\Lambda}$ extends to an automorphism of $B^{\Lambda}$ and produces a dynamical system $\left(B^{\Lambda}, \mathbb{Z}^{k}, \alpha^{\Lambda}\right)$. The action $\alpha^{\Lambda}$ is called the dual action, see [39, p. 190].

Takai duality implies that $C^{*}(\Lambda)$ is isomorphic to a full corner of $B^{\Lambda} \times_{\alpha^{\Lambda}} \mathbb{Z}^{k}$. Specifically, there is an inclusion map $\theta_{\Lambda}: C^{*}(\Lambda) \rightarrow B^{\Lambda} \times_{\alpha^{\Lambda}} \mathbb{Z}^{k}$, which is given by $\theta_{\Lambda}\left(s_{\lambda}\right):=$ $i_{B^{\Lambda}}\left(i_{\mathbb{T}}(1) i_{C^{*}(\Lambda)}\left(s_{\lambda}\right)\right) i_{\mathbb{Z}^{k}}(d(\lambda))^{*}$, and which induces the identity map in $K$-theory].

Lemma 4.5. Let $\Lambda$ be a row finite $k$-graph with no sources, $H \subsetneq \Lambda^{0}$ a hereditary saturated subset of $\Lambda^{0}$ and $\Gamma=H \Lambda$. There is a homomorphism $\varphi: C^{*}(\Gamma) \rightarrow C^{*}(\Lambda)$ satisfying

$$
\varphi\left(s_{\lambda}\right)=s_{\lambda} \quad \text { for each } \quad \lambda \in \Gamma \text {. }
$$

\footnotetext{
${ }^{1}$ This is discussed in detail in terms of skew-product graphs in [24, Lemma 5.2]; to translate back to the language of crossed-products, recall that there is an isomorphism $C^{*}(\Lambda) \times_{\gamma} \mathbb{T}^{k} \cong C^{*}\left(\Lambda \times_{d} \mathbb{Z}^{k}\right)$ that carries each $i_{C^{*}(\Lambda)}\left(s_{\lambda}\right) i_{\mathbb{T}^{k}}\left(z^{n}\right)$ to $s_{(\lambda, n)}$ [21, Corollary 5.3].
} 
This $\varphi$ extends to a homomorphism $\hat{\varphi}: B^{\Gamma} \times_{\alpha^{\Gamma}} \mathbb{Z}^{k} \rightarrow B^{\Lambda} \times_{\alpha^{\Lambda}} \mathbb{Z}^{k}$ such that the following diagram commutes:

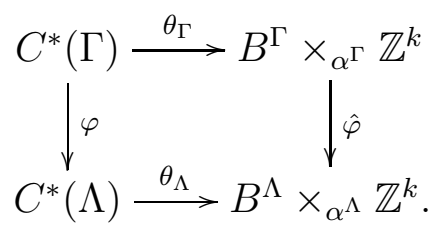

Proof. Lemma 3.7 gives a homomorphism $\varphi: C^{*}(\Gamma) \rightarrow C^{*}(\Lambda)$ satisfying (4.2). This $\varphi$ is equivariant for the gauge actions on $C^{*}(\Gamma)$ and $C^{*}(\Lambda)$, so it induces a homomorphism $\varphi \times$ id : $B^{\Gamma} \rightarrow B^{\Lambda}$ mapping $C_{c}\left(\mathbb{T}^{k}, C^{*}(\Gamma)\right)$ to $C_{c}\left(\mathbb{T}^{k}, C^{*}(\Lambda)\right)$ via $\varphi \times \operatorname{id}(f)(z)=\varphi(f(z))$, see [39, Corollary 2.48].

By construction, we have $(\varphi \times \mathrm{id}) \circ i_{C^{*}(\Gamma)}\left(s_{\lambda}\right)=i_{C^{*}(\Lambda)} \circ \varphi\left(s_{\lambda}\right)$ for every $\lambda \in \Gamma$. The commutativity of the diagram

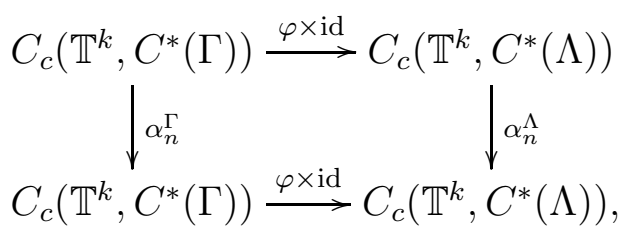

shows that $\varphi \times$ id is equivariant for the dual actions of $\mathbb{Z}^{k}$ on $B^{\Gamma}$ and $B^{\Lambda}$. Hence $\varphi \times$ id induces a homomorphism $\hat{\varphi}: B^{\Gamma} \times_{\alpha^{\Gamma}} \mathbb{Z}^{k} \rightarrow B^{\Lambda} \times_{\alpha^{\Lambda}} \mathbb{Z}^{k}$. Using the description of $\theta_{\Lambda}$ and $\theta_{\Gamma}$ above, we see that the diagram (4.3) commutes.

Following [23] a homology spectral sequence $\left(E^{r}, d^{r}, \alpha^{r}\right)$ or simply $\left(E^{r}, d^{r}\right)$ consists of the following data:

(i) A family of $\mathbb{Z}$-modules $E_{a, b}^{r}$ defined for all integers $a, b$ and $r>0$.

(ii) Maps $d_{a, b}^{r}: E_{a, b}^{r} \rightarrow E_{a-r, b+r-1}^{r}$ that are differentials, i.e, $d_{a-r, b+r-1}^{r} \circ d_{a, b}^{r}=0$

(iii) Maps $\alpha_{a, b}^{r}: E_{a, b}^{r+1} \rightarrow H\left(E_{a, b}^{r}\right)$ that are isomorphisms, where $H\left(E_{a, b}^{r}\right)$ denotes the module $\operatorname{ker}\left(d_{a, b}^{r}\right) / \operatorname{im}\left(d_{a+r, b-r+1}^{r}\right)$.

A morphism of spectral sequences $f:\left(E^{r}, d^{r}\right) \rightarrow\left(\tilde{E}^{r}, \tilde{d}^{r}\right)$ is a family of morphisms $f^{r}: E^{r} \rightarrow \tilde{E}^{r}$ such that (1) the morphisms $f^{r}$ commute with the differentials, and (2) the induced homomorphisms $H\left(f_{a, b}^{r}\right): H\left(E_{a, b}^{r}\right) \rightarrow H\left(\tilde{E}_{a, b}^{r}\right)$ make the following diagram commute:

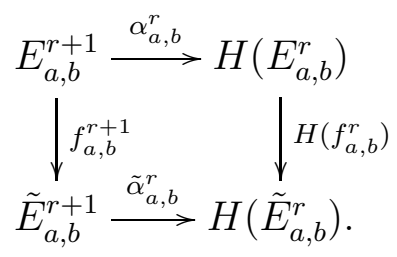

Recall that the spectral sequence $E_{a, b}^{r}$ is bounded if for each $r$ and $n$ there are just finitely many nonzero terms $E_{a, b}^{r}$ such that $a+b=n$ (note that this holds for all $r$ if it holds for $r=1$ ). We then eventually have $E_{a, b}^{r+1} \cong E_{a, b}^{r}$ for all $a, b$, and we write $E_{a, b}^{\infty}$ for this limiting term. We say that the spectral sequence $E_{a, b}^{r}$ converges to a sequence $\mathcal{K}_{*}=\left\{\mathcal{K}_{n}: n \in \mathbb{Z}\right\}$ of modules if each $\mathcal{K}_{n}$ admits a finite filtration $0=F_{s}\left(\mathcal{K}_{n}\right) \subseteq F_{s+1}\left(\mathcal{K}_{n}\right) \subseteq \cdots \subseteq F_{t}\left(\mathcal{K}_{n}\right)=\mathcal{K}_{n}$ such that $E_{a, b}^{\infty} \cong F_{a}\left(\mathcal{K}_{a+b}\right) / F_{a-1}\left(\mathcal{K}_{a+b}\right)$ for $s<a \leq t$. 
The following result essentially follows from the argument of [10, Theorem 3.15] (though we are following the notation of [23, Proposition 5.4 and Theorem 5.5]), but we need a more detailed statement.

Lemma 4.6. Let $\Lambda, \Gamma$ and $\hat{\varphi}$ be as in Lemma 4.5. There exist spectral sequences $\left(E^{r}, d^{r}\right)$ and $\left(\tilde{E}^{r}, \tilde{d}^{r}\right)$ and a morphism of spectral sequences $f:\left(E^{r}, d^{r}\right) \rightarrow\left(\tilde{E}^{r}, \tilde{d}^{r}\right)$ such that

(a) $\left(E^{r}, d^{r}\right)$ converges to $K_{*}\left(B^{\Gamma} \times_{\alpha^{\Gamma}} \mathbb{Z}^{k}\right)$ and $\left(\tilde{E}^{r}, \tilde{d}^{r}\right)$ converges to $K_{*}\left(B^{\Lambda} \times_{\alpha^{\Lambda}} \mathbb{Z}^{k}\right)$,

(b) $E_{a, b}^{1}=D_{a}^{\Gamma}$ and $\tilde{E}_{a, b}^{1}=D_{a}^{\Lambda}$ for $0 \leq a \leq k$ and $b$ even,

(c) $d_{a, b}^{1}=\partial_{a}^{\Gamma}$ and $\tilde{d}_{a, b}^{1}=\partial_{a}^{\Lambda}$ for $1 \leq a \leq k$ and $b$ even,

(d) the isomorphisms $E_{a, b}^{\infty} \cong K_{*}\left(B^{\Gamma} \times_{\alpha^{\Gamma}} \mathbb{Z}^{k}\right)$ and $\tilde{E}_{a, b}^{\infty} \cong K_{*}\left(B^{\Lambda} \times_{\alpha^{\Lambda}} \mathbb{Z}^{k}\right)$ of part (a) intertwine the morphisms $f_{a, b}^{\infty}: E_{a, b}^{\infty} \rightarrow \tilde{E}_{a, b}^{\infty}$ and the homomorphisms $K_{*}(\hat{\varphi})$ : $K_{*}\left(B^{\Gamma} \times_{\alpha^{\Gamma}} \mathbb{Z}^{k}\right) \rightarrow K_{*}\left(B^{\Lambda} \times_{\alpha^{\Lambda}} \mathbb{Z}^{k}\right)$, and

(e) $f_{a, b}^{1}: D_{a}^{\Gamma} \rightarrow D_{a}^{\Lambda}$ coincides with the map $j_{a}^{H}$ of Example 4.2.

Proof. Firstly we briefly recall parts of the proof of [23, Theorem 5.5] that we will need. Since the vertex projections $s_{v} \in C^{*}(\Lambda)$ are fixed by the gauge action, the map that sends $a \in C^{*}(\Lambda)$ to the constant function $z \mapsto a \in C\left(\mathbb{T}^{k}, C^{*}(\Lambda)\right)$ determines a homomorphism $\varepsilon^{\Lambda}: C_{0}\left(\Lambda^{0}\right) \cong C^{*}\left(\left\{s_{v}: v \in \Lambda^{0}\right\}\right) \rightarrow B^{\Lambda}$; so $\varepsilon^{\Lambda}\left(\delta_{v}\right)$ is the constant function $z \mapsto s_{v}$ on $\mathbb{T}^{k}$. Let $\varepsilon_{*}^{\Lambda}: \mathbb{Z} \Lambda^{0} \rightarrow K_{0}\left(B^{\Lambda}\right)$ be the induced map in $K_{0}$. The map

$$
\operatorname{id} \otimes \varepsilon_{*}^{\Lambda}: \bigwedge^{*} \mathbb{Z}^{k} \otimes \mathbb{Z} \Lambda^{0} \rightarrow \bigwedge^{*} \mathbb{Z}^{k} \otimes K_{0}\left(B^{\Lambda}\right)
$$

is a map of complexes that induces an isomorphism on homology [10, Theorem 3.14]. Thus, as in [10, 23],

$$
H_{*}\left(\mathbb{Z}^{k}, K_{0}\left(B^{\Lambda}\right)\right) \cong H_{*}\left(\bigwedge^{*} \mathbb{Z}^{k} \otimes \mathbb{Z} \Lambda^{0}\right) .
$$

Therefore setting $\tilde{E}_{a, b}^{1}=D_{a}^{\Lambda}$ for $0 \leq a \leq k$ and $b$ even, and $\tilde{d}_{a, b}^{1}=\partial_{a}^{\Lambda}$ for $1 \leq a \leq k$ and $b$ even (and zero otherwise), yields

$$
\tilde{E}_{a, b}^{2} \cong \begin{cases}H_{a}\left(\mathbb{Z}^{k}, K_{0}\left(B^{\Lambda}\right)\right) & \text { if } 0 \leq a \leq k \text { and } b \text { even } \\ 0 & \text { otherwise. }\end{cases}
$$

It follows that the spectral sequence $\left(\tilde{E}^{r}, \tilde{d}^{r}\right)$ converges to $K_{*}\left(B^{\Lambda} \times_{\alpha^{\Lambda}} \mathbb{Z}^{k}\right)$ as required.

We now diverge slightly from the proof of [23, Theorem 5.5] and apply Lemma 4.5: Fix a hereditary saturated set $H \subsetneq \Lambda^{0}$, and let $\Gamma=H \Lambda$. Repeating the previous argument on $\Gamma$ (using $E$ rather than $\tilde{E}$ for the new sequence) we get spectral sequences $\left(E^{r}, d^{r}\right)$, $\left(\tilde{E}^{r}, \tilde{d}^{r}\right)$ satisfying properties (a)-(c).

Define $\varepsilon_{*}^{\Gamma}$ in the same way as $\varepsilon_{*}^{\Lambda}$ and let

$$
\iota: C_{0}\left(\Gamma^{0}\right) \rightarrow C_{0}\left(\Lambda^{0}\right)
$$

be the extension map extending by zero. Using Lemma 4.5 and the canonical identifications $K_{0}\left(C_{0}\left(\Gamma^{0}\right)\right) \cong \mathbb{Z} \Gamma^{0}$ and $K_{0}\left(C_{0}\left(\Lambda^{0}\right)\right) \cong \mathbb{Z} \Lambda^{0}$, we have the commuting diagram below:

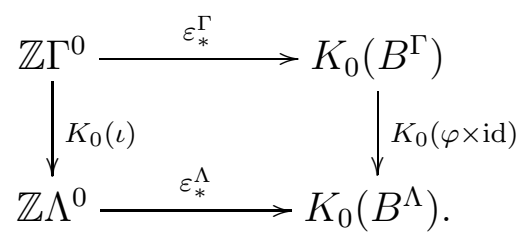


Taking the tensor product of the chain complex $\wedge^{*} \mathbb{Z}^{k}$ with the groups appearing in (4.5) and applying the homology functor, we get

$$
\begin{aligned}
& H_{*}\left(D_{*}^{\Gamma}\right)=H_{*}\left(\bigwedge^{*} \mathbb{Z}^{k} \otimes \mathbb{Z} \Gamma^{0}\right) \stackrel{H_{*}\left(\mathrm{id} \otimes \varepsilon_{*}^{\Gamma}\right)}{\longrightarrow} H_{*}\left(\bigwedge^{*} \mathbb{Z}^{k} \otimes K_{0}\left(B^{\Gamma}\right)\right) \\
& \downarrow_{H_{*}\left(\mathrm{id} \otimes K_{0}(\iota)\right)} \quad H_{*}\left(\mathrm{id} \otimes K_{0}(\varphi \times \mathrm{id})\right) \\
& H_{*}\left(D_{*}^{\Lambda}\right)=H_{*}\left(\bigwedge^{*} \mathbb{Z}^{k} \otimes \mathbb{Z} \Lambda^{0}\right) \stackrel{H_{*}\left(\mathrm{id} \otimes \varepsilon_{*}^{\Lambda}\right)}{\longrightarrow} H_{*}\left(\bigwedge^{*} \mathbb{Z}^{k} \otimes K_{0}\left(B^{\Lambda}\right)\right) .
\end{aligned}
$$

As in the displayed equation at the top of [23, p. 187] we have isomorphisms $H_{*}\left(\bigwedge^{*} \mathbb{Z}^{k} \otimes\right.$ $\left.K_{0}\left(B^{\Gamma}\right)\right) \rightarrow H_{*}\left(\mathbb{Z}^{k}, K_{0}\left(B^{\Gamma}\right)\right)$ and $H_{*}\left(\bigwedge^{*} \mathbb{Z}^{k} \otimes K_{0}\left(B^{\Lambda}\right)\right) \rightarrow H_{*}\left(\mathbb{Z}^{k}, K_{0}\left(B^{\Lambda}\right)\right)$ such that the following diagram commutes

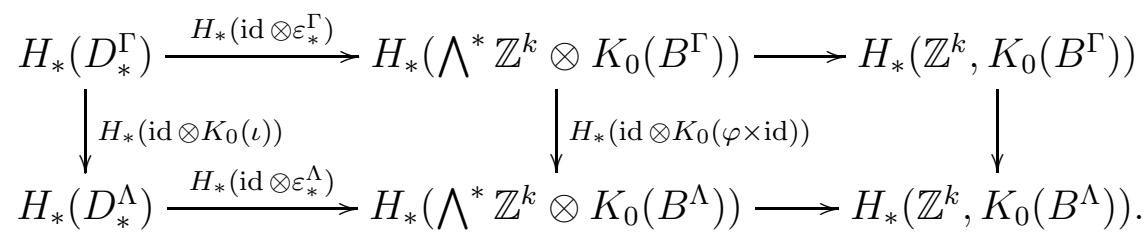

The composition of the horizontal maps in the diagram (4.6) is the isomorphism described by Evans in [10, Theorem 3.14]. Hence $H_{*}\left(\mathrm{id} \otimes \varepsilon_{*}^{\Gamma}\right)$ and $H_{*}\left(\mathrm{id} \otimes \varepsilon_{*}^{\Lambda}\right)$ are isomorphisms. Thus the left-hand vertical map on homology (induced by id $\left.\otimes K_{0}(\iota)\right)$ coincides with the right-hand map (induced by id $\otimes K_{0}(\varphi \times$ id)).

Applying the naturality of [23, Proposition 5.4] yields a morphism $f$ of spectral sequences compatible with $K_{*}(\hat{\varphi}): K_{*}\left(B^{\Gamma} \times_{\alpha^{\Gamma}} \mathbb{Z}^{k}\right) \rightarrow K_{*}\left(B^{\Lambda} \times_{\alpha^{\Lambda}} \mathbb{Z}^{k}\right)$ such that $f_{a, b}^{1}: D_{a}^{\Gamma} \rightarrow$ $D_{a}^{\Lambda}$ is given by id $\otimes K_{0}(\iota)$. The latter map agrees with $j_{a}^{H}$ from Example 4.2 giving (d)-(e) as required.

Recall that a $C^{*}$-algebra $A$ is $K_{0}$-liftable if for every pair of ideals $I \subseteq J$ in $A$, the extension

$$
0 \longrightarrow I \stackrel{\varphi}{\longrightarrow} J \stackrel{\pi}{\longrightarrow} J / I \longrightarrow 0
$$

has the property that the induced map $K_{0}(\pi): K_{0}(J) \rightarrow K_{0}(J / I)$ is surjective, or equivalently that $K_{1}(\varphi): K_{1}(I) \rightarrow K_{1}(J)$ is injective (see [31, Definition 3.1]). In fact, it suffices to consider $J=A$, a fact that the authors of [31] attribute to Larry Brown. So $A$ is $K_{0}$-liftable if $K_{1}(\varphi): K_{1}(I) \rightarrow K_{1}(A)$ is injective for every ideal $I$ in $A$.

The next lemma allows us to relate $K_{0}$-liftability of $C^{*}(\Lambda)$ to the homology of the complex $D^{\Lambda}$ described earlier.

Lemma 4.7. Let $\Lambda$ be a row-finite 2-graph with no sources. Suppose that $H \subseteq \Lambda^{0}$ is saturated and hereditary. Let $j^{H}: D_{*}^{\Gamma} \rightarrow D_{*}^{\Lambda}$ be the map of complexes induced by the inclusion $\Gamma^{0}=H \hookrightarrow \Lambda^{0}$, and let $\varphi: C^{*}(\Gamma) \rightarrow C^{*}(\Lambda)$ be the inclusion that carries a generator $s_{\lambda}$ of $C^{*}(\Gamma)$ to the corresponding generator $s_{\lambda}$ of $C^{*}(\Lambda)$. Then there are isomorphisms $K_{1}\left(C^{*}(\Gamma)\right) \cong H_{1}\left(D^{\Gamma}\right)$ and $K_{1}\left(C^{*}(\Lambda)\right) \cong H_{1}\left(D^{\Lambda}\right)$ making the following diagram commute:

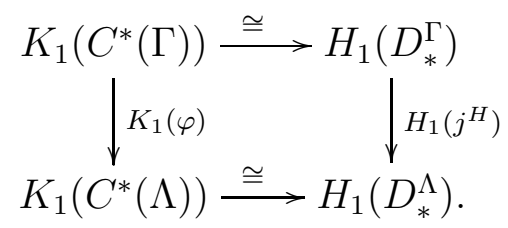

In particular, $K_{1}(\varphi)$ is injective if and only if $H_{1}\left(j^{H}\right)$ is injective. 
Proof. Define $\Gamma=H \Lambda$. By Lemma 4.6 we have the spectral sequence $\left(\tilde{E}^{r}, \tilde{d}^{r}\right)$ converging to $K_{0}\left(B^{\Lambda} \times_{\alpha^{\Lambda}} \mathbb{Z}^{2}\right)$. Since $\Lambda$ is a 2 -graph and by the convergence from Lemma 4.6)(a) we have the following filtration of $\tilde{\mathcal{K}}_{1}=K_{1}\left(B^{\Lambda} \times_{\alpha^{\Lambda}} \mathbb{Z}^{2}\right)$ :

$$
0=F_{0}\left(\tilde{\mathcal{K}}_{1}\right) \subseteq F_{1}\left(\tilde{\mathcal{K}}_{1}\right) \subseteq F_{2}\left(\tilde{\mathcal{K}}_{1}\right)=\tilde{\mathcal{K}}_{1},
$$

with $F_{1}\left(\tilde{\mathcal{K}}_{1}\right) \cong \tilde{E}_{1,0}^{2}, F_{2}\left(\tilde{\mathcal{K}}_{1}\right) \cong \tilde{\mathcal{K}}_{1}$ and $F_{2}\left(\tilde{\mathcal{K}}_{1}\right) / F_{1}\left(\tilde{\mathcal{K}}_{1}\right)=\{0\}$ (see [10]). The same applies to the convergent spectral sequence $\left(E^{r}, d^{r}\right)$. We let $\mathcal{K}_{*}=K_{*}\left(B^{\Gamma} \times_{\alpha^{\Gamma}} \mathbb{Z}^{2}\right)$ denote its limit. Since $f$ is compatible with $K_{*}(\hat{\varphi}): \mathcal{K}_{*} \rightarrow \tilde{\mathcal{K}}_{*}$ by Lemma 4.6(d), we have the commuting diagram

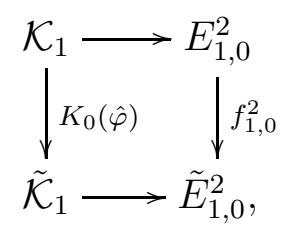

and the horizontal maps are isomorphisms. Using that $\tilde{E}_{1,0}^{2} \cong H\left(\tilde{E}_{1,0}^{1}\right)=H_{1}\left(D_{*}^{\Lambda}\right)$ and $E_{1,0}^{2} \cong H\left(E_{1,0}^{1}\right)=H_{1}\left(D_{*}^{\Gamma}\right)$ we obtain the following commuting diagram:

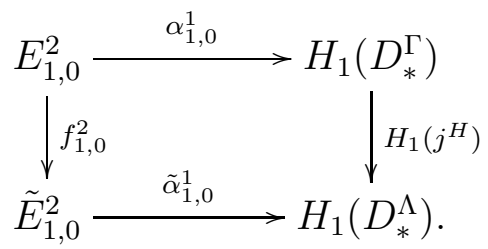

Applying the $K_{1}$-functor to the commuting diagram

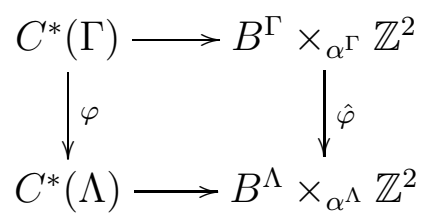

of Lemma 4.5 we get a commuting diagram in which the horizontal maps are invertible. Combining this and that $\tilde{\mathcal{K}}_{1}=K_{1}\left(B^{\Lambda} \times_{\alpha^{\Lambda}} \mathbb{Z}^{2}\right)$ and $\mathcal{K}_{1}=K_{1}\left(B^{\Gamma} \times_{\alpha^{\Gamma}} \mathbb{Z}^{2}\right)$ with the previous diagrams we obtain the commuting diagram

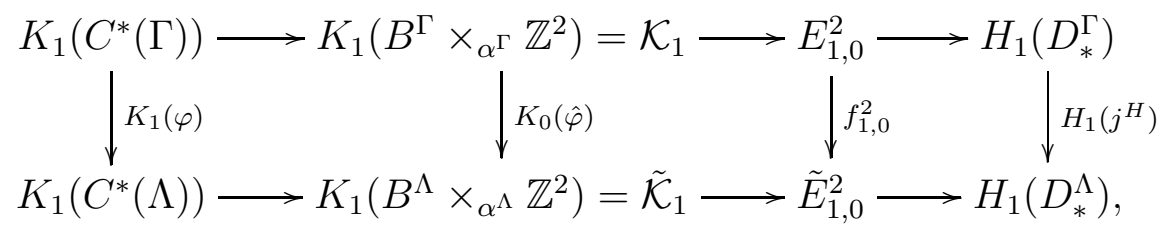

where the horizontal maps are all isomorphisms. The final statement follows immediately.

Proof of Theorem 4.3. We start by proving (2), so assume that $C^{*}(\Lambda)$ has real-rank zero. Lemma 4.1 implies that $\Lambda$ is strongly aperiodic. As alluded to immediately after [31, Definition 3.1], all $C^{*}$-algebras of real rank zero are $K_{0}$-liftable: Theorem 2.10 of [4] shows that if $A$ has real-rank zero, then so does every $M_{n}(A)$, and then applying [4, Theorem 3.14] to each $M_{n}(A)$ shows that if $I$ is an ideal of $A$ then every projection in $M_{n}(A / I)$ lifts to a projection in $M_{n}(A)$. Since $A$, and hence also $A / I$, has real-rank zero, 
$K_{0}(A / I)$ is generated by classes of projections over $A / I$, and it follows that $A$ is $K_{0^{-}}$ liftable. Hence every inclusion $I \subseteq C^{*}(\Lambda)$ of a proper ideal induces an injective map on $K_{1}$-groups. In particular, for every hereditary saturated subset $H \subseteq \Lambda^{0}$, the map $H_{1}\left(j^{H}\right)$ is injective by Lemma 4.7 .

We now prove (1). Suppose that $C^{*}(\Lambda)$ is purely infinite. The preceding paragraph establishes (i) $\Rightarrow$ (ii). To prove the reverse implication (ii) $\Rightarrow$ (i) suppose that $\Lambda$ is strongly aperiodic and that $H_{1}\left(j^{H}\right)$ is injective for each $H$. Theorem 3.2 implies that $C^{*}(\Lambda)$ has topological dimension zero. So [31, Theorem 4.2] implies that $C^{*}(\Lambda)$ has real-rank zero if and only if it is $K_{0}$-liftable, and it suffices to establish the latter.

Fix a non-trivial ideal $I$ of $C^{*}(\Lambda)$. We need to show that the inclusion $\iota: I \hookrightarrow C^{*}(\Lambda)$ induces an injective map at the level of $K_{1}$-groups. Since $\Lambda$ is strongly aperiodic it follows from Corollary 3.9 that $I=I_{H}$ for some nonempty hereditary saturated $H \subsetneq \Lambda^{0}$. Let $\Gamma:=H \Lambda$. As in Lemma 3.7, the $C^{*}$-algebra $C^{*}(\Gamma)$ is isomorphic to a full corner of $I$, and so the inclusion map $C^{*}(\Gamma) \hookrightarrow I$ induces an isomorphism in $K$-theory. We obtain the following commuting diagram:

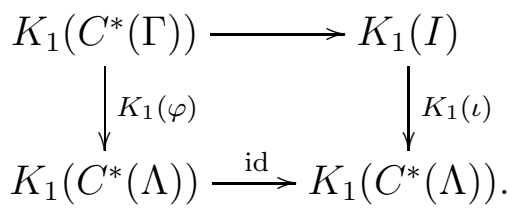

So $K_{1}(\iota)$ is injective if and only if $K_{1}(\varphi)$ is injective. By condition (ii), the map $H_{1}\left(j^{H}\right)$ is injective, and so Lemma 4.7 shows that $K_{1}(\varphi)$ is injective as required.

To finish off the section, we show how to reduce checking real-rank zero for 2-graph algebras with finite ideal lattice to checking real-rank zero for simple 2-graph algebras, without any assumption of pure infiniteness. We thank an anonymous referee for pointing out this application of our arguments.

Proposition 4.8. Let $\Lambda$ be a row-finite 2-graph with no sources, and suppose that $C^{*}(\Lambda)$ has finite ideal lattice. Then $\Lambda$ is strongly aperiodic, and the following are equivalent:

(1) $C^{*}(\Lambda)$ has real-rank zero;

(2) Both of the following hold:

(i) for every saturated hereditary $H \subseteq \Lambda^{0}$, the map $H_{1}\left(j^{H}\right)$ of Example 4.2 is injective; and

(ii) for every pair $K \subseteq H$ where $K$ is a saturated hereditary subset of $\Lambda^{0}$ and $H \backslash K$ is a saturated hereditary subset of $\Lambda \backslash \Lambda K$ such that $H \Lambda \backslash \Lambda K$ is cofinal, the $C^{*}$-algebra $C^{*}(H \Lambda \backslash \Lambda K)$ has real-rank zero.

Proof. Every $C^{*}$-algebra with finite ideal lattice has topological dimension zero, and so Theorem 3.2 shows that $\Lambda$ is strongly aperiodic.

First suppose that $C^{*}(\Lambda)$ has real-rank zero. Then part (2) of Theorem 4.3 gives $(2 \mathrm{i})$. Fix a nested pair $K \subseteq H$ of saturated hereditary sets such that $H \Lambda \backslash \Lambda K$ is cofinal. Then $C^{*}(\Lambda \backslash \Lambda H)$ is a quotient of $C^{*}(\Lambda)$ by [32, Theorem 5.2(b)] and therefore has real-rank zero. Moreover, $C^{*}(H \Lambda \backslash \Lambda K)$ is isomorphic to a full corner of an ideal in $C^{*}(\Lambda \backslash \Lambda K)$ by [32, Theorem 5.2(c)], and therefore itself also has real-rank zero, giving (2ii).

Now suppose that $(2)$ holds. We prove that $C^{*}(\Lambda)$ has real-rank zero by induction on $\left|\operatorname{Prim} C^{*}(\Lambda)\right|$. If Prim $C^{*}(\Lambda)$ is a singleton, then $\Lambda$ itself is cofinal [21, Proposition 4.8], so we can take $K=\emptyset$ and $H=\Lambda^{0}$ in (2) to see that $C^{*}(\Lambda)=C^{*}(H \Lambda \backslash \Lambda K)$ has real-rank 
zero by hypothesis. Now fix $\Lambda$ and suppose that our assertion holds for every strongly aperiodic $k$-graph with fewer primitive ideals than $C^{*}(\Lambda)$. Since $\operatorname{Prim} C^{*}(\Lambda)$ is finite, we can fix an ideal $I$ of $C^{*}(\Lambda)$ such that $C^{*}(\Lambda) / I$ is simple. Since $\Lambda$ is strongly aperiodic, $I$ is generated by $\left\{p_{v}: v \in K\right\}$ for some saturated hereditary $K \subseteq \Lambda^{0}$. We then have $C^{*}(\Lambda) / I \cong C^{*}(\Lambda \backslash \Lambda K)$ by [32, Theorem 5.2(b)] again, and since this $C^{*}$-algebra is simple, $\Lambda \backslash \Lambda K$ is cofinal. So $(2 \mathrm{ii})$ with $H=\Lambda^{0}$ says that $C^{*}(\Lambda) / I$ has real-rank zero. Lemma 4.7 and $(2 \mathrm{i})$ show that $C^{*}(\Lambda)$ is $K_{0}$-liftable, and it follows from [4, Proposition 3.15] that if $I$ has real-rank zero, then every projection in $C^{*}(\Lambda) / I$ lifts to a projection in $C^{*}(\Lambda)$. Therefore, by [4, Theorem 3.14], to prove that $C^{*}(\Lambda)$ has real-rank zero, it now suffices to prove that $I$ has real-rank zero.

Consider the $k$-graph $\Gamma:=K \Lambda$. By Lemma 3.7, $C^{*}(\Gamma)$ is isomorphic to a full corner of $I$, so [4, Theorem 3.8] implies that it suffices to prove that $C^{*}(\Gamma)$ has real-rank zero. Since $C^{*}(\Lambda)$ is $K_{0}$-liftable, so is $I$, and hence also $C^{*}(\Gamma)$ since the inclusion map $C^{*}(\Gamma) \hookrightarrow I$ induces an isomorphism in $K$-theory. The $k$-graph $\Gamma$ is strongly aperiodic because $\Lambda$ is strongly aperiodic. Suppose that $K^{\prime}$ and $H^{\prime}$ are saturated hereditary subsets of $\Gamma^{0}$ as in (2ii) for $\Gamma$; so $H^{\prime} \backslash K^{\prime}$ is saturated and hereditary in $\Gamma \backslash \Gamma K^{\prime}$, and $H^{\prime} \Gamma \backslash \Gamma K^{\prime}$ is cofinal. Then $H^{\prime}$ and $K^{\prime}$ also satisfy these hypotheses with respect to $\Lambda$ (because $\Gamma^{0}=K$ is saturated in $\Lambda)$. Hence hypothesis (2ii) for $C^{*}(\Lambda)$ implies that $C^{*}\left(H^{\prime} \Gamma \backslash \Gamma K^{\prime}\right)=$ $C^{*}\left(H^{\prime} \Lambda \backslash \Lambda K^{\prime}\right)$ has real-rank zero. Thus $\Gamma$ satisfies (2i) and (2ii). By construction, $C^{*}(\Gamma)=C^{*}(\Lambda) / I$ has fewer primitive ideals than $C^{*}(\Lambda)$, and so the inductive hypothesis implies that $C^{*}(\Gamma)$ has real-rank zero as required.

\section{Purely infinite $k$-Graph $C^{*}$-Algebras}

In this section we turn our attention to pure infiniteness of higher-rank graph $C^{*}$ algebras, which is one of the assumptions in Theorem 4.3. In [19], Kirchberg and Rørdam introduced three separate notions of purely infinite $C^{*}$-algebras; weakly purely infinite, purely infinite and strongly purely infinite. As the names suggest, strong pure infiniteness implies pure infiniteness which implies weak pure infiniteness. Of these notions, strong pure infiniteness is perhaps the most useful in the classification of non-simple $C^{*}$-algebras. Indeed, Kirchberg showed in [17] that two separable, nuclear, stable, strongly purely infinite $C^{*}$-algebras with the same primitive ideal space $X$ are isomorphic if and only if they are $K K_{X}$-equivalent.

Following [18, we denote the set of positive elements in a $C^{*}$-algebra $A$ by $A^{+}$. The ideal in $A$ generated by an element $b$ is denoted $\overline{A b A}$. Recall that for positive elements $a \in M_{n}(A)$ and $b \in M_{m}(A)$, we say that $a$ is Cuntz below $b$, denoted $a \precsim b$, if there exists a sequence of elements $x_{k}$ in $M_{m, n}(A)$ such that $x_{k}^{*} b x_{k} \rightarrow a$ in norm. We say $A$ is purely infinite if there are no characters on $A$ and for all $a, b \in A^{+}$, we have $a \precsim b$ if and only if $a \in \overline{A b A}$ (see [18, Definition 4.1]).

Let $\Lambda$ be a 2-graph and $u \in \Lambda^{0}$. As introduced in [16], given $a, b \in \mathbb{Z}^{+}$, an $(a, b)$-aperiodic quartet, or just an aperiodic quartet, at $u$ consists of distinct paths $\alpha_{1}, \alpha_{2} \in u \Lambda^{a e_{1}} u$ and distinct paths $\beta_{1}, \beta_{2} \in u \Lambda^{b e_{2}} u$ such that $\beta_{2} \alpha_{1}=\alpha_{1} \beta_{2}, \beta_{2} \alpha_{2}=\alpha_{2} \beta_{2}, \beta_{1} \alpha_{1}=\alpha_{2} \beta_{1}$, and $\beta_{1} \alpha_{2}=\alpha_{1} \beta_{1}$.

Proposition 5.1. Let $\Lambda$ be a row-finite 2-graph with no sources. Suppose that there is an aperiodic quartet at $u$ for each $u \in \Lambda^{0}$. Then $C^{*}(\Lambda)$ is strongly purely infinite.

Before giving a proof of Proposition 5.1 we present a characterisation of when the $C^{*}$-algebra $C^{*}(\Lambda)$ of a row-finite strongly aperiodic $k$-graph $\Lambda$ with no sources is purely 
infinite. Recall that a projection $p$ in a $C^{*}$-algebra $A$ is infinite if it is Murray-von Neumann equivalent to a proper subprojection of itself, and properly infinite if there are two mutually orthogonal subprojections of $p$ in $A$, each one Murray-von Neumann equivalent to $p$.

Lemma 5.2. Let $\Lambda$ be a row-finite $k$-graph with no sources. Suppose that $\Lambda$ is strongly aperiodic. Then the following are equivalent

(i) The $C^{*}$-algebra $C^{*}(\Lambda)$ is purely infinite.

(ii) For every $v \in \Lambda^{0}$ the projection $s_{v}$ is properly infinite in $C^{*}(\Lambda)$.

(iii) For every hereditary saturated $H \subseteq \Lambda^{0}$ and every $v \in \Lambda^{0} \backslash H$, the projection $s_{v}$ is infinite in $C^{*}(\Lambda \backslash \Lambda H)$.

(iii') For every hereditary saturated $H \subseteq \Lambda^{0}$ and every $v \in \Lambda^{0} \backslash H$, the projection $s_{v}$ is properly infinite in $C^{*}(\Lambda \backslash \Lambda H)$.

(iv) For every hereditary saturated $H \subseteq \Lambda^{0}$ and every $v \in \Lambda^{0} \backslash H$, the $C^{*}$-algebra $s_{v} C^{*}(\Lambda \backslash \Lambda H) s_{v}$ contains an infinite projection.

(iv') For every hereditary saturated $H \subseteq \Lambda^{0}$ and every $v \in \Lambda^{0} \backslash H$, the $C^{*}$-algebra $s_{v} C^{*}(\Lambda \backslash \Lambda H) s_{v}$ contains a properly infinite projection.

(v) Every non-zero hereditary sub-C $C^{*}$-algebra in any quotient of $C^{*}(\Lambda)$ contains an infinite projection.

$\left(\mathrm{v}^{\prime}\right)$ Every non-zero hereditary sub- $C^{*}$-algebra in any quotient of $C^{*}(\Lambda)$ contains a properly infinite projection.

Proof of Lemma 5.2. We first prove (i) $\Leftrightarrow($ ii) $\Leftrightarrow($ iii $) \Leftrightarrow($ iv $) \Leftrightarrow(\mathrm{v})$.

The implication (i) $\Rightarrow$ (ii) is known, see [18, Theorem 4.16]. For (ii) $\Rightarrow$ (iii), observe that [32, Theorem 5.2] shows that $C^{*}(\Lambda \backslash \Lambda H)$ is isomorphic to a quotient of $C^{*}(\Lambda)$, and that the quotient map carries $s_{v}$ to zero if and only if $v \in H$. Corollary 3.15 of [18] says that the image of a properly infinite projection under a $C^{*}$-homomorphism is either zero or infinite, giving (ii) $\Rightarrow$ (iii). The implication (iii) $\Rightarrow$ (iv) is trivial because $s_{v} \in s_{v} C^{*}(\Lambda \backslash \Lambda H) s_{v}$ for each $v \in \Lambda^{0} \backslash H$.

For (iv) $\Rightarrow(\mathrm{v})$, fix a proper ideal $I$ of $C^{*}(\Lambda)$. Let $B$ be a non-zero hereditary subalgebra of $C^{*}(\Lambda) / I$. By Corollary [3.9] and [32, Theorem 5.2], there is a saturated hereditary subset $H \subseteq \Lambda^{0}$ such that $\Gamma:=\Lambda \backslash \Lambda H$ satisfies $C^{*}(\Lambda) / I \cong C^{*}(\Gamma)$. So $B$ is isomorphic to a hereditary subalgebra of $C^{*}(\Gamma)$. We identify the two henceforth.

Select any non-zero positive element $a \in B$. Since $\Lambda$ is strongly aperiodic, Theorem 3.9 implies that $\mathcal{G}_{\Gamma}$ is topologically principal. Hence [2, Lemma 3.2] provides a non-zero positive element $h \in C^{*}\left(\left\{s_{\lambda} s_{\lambda}^{*}: \lambda \in \Gamma\right\}\right) \cong C_{0}\left(\mathcal{G}_{\Gamma}^{(0)}\right)$ such that $h \precsim a$. Recall that the cylinder sets $\{Z(\lambda): \lambda \in \Gamma\}$ form a basis for the topology on $\mathcal{G}_{\Lambda}^{(0)}$ (see [21, Definition 2.4]). Choose $\lambda \in \Gamma$ such that $h(x) \geq\|h\| / 2>0$ for all $x \in Z(\lambda)$, and let $v:=s(\lambda) \in \Gamma^{0}$. Let $g \in C_{0}\left(\mathcal{G}_{\Gamma}^{(0)}\right)$ be the function $g(x):=h(x)^{-1 / 2}$ for $x \in Z(\lambda)$ and zero otherwise. Then $g h g=1_{Z(\lambda)}=s_{\lambda} s_{\lambda}^{*}$ and $\left(g s_{\lambda}\right)^{*} h g s_{\lambda}=s_{\lambda}^{*} s_{\lambda} s_{\lambda}^{*} s_{\lambda}=s_{v}$. In particular, $s_{v} \precsim h$ (take $x_{k}:=g s_{\lambda}$ for each $k$ ). By assumption (iv) there is an infinite projection $p \in s_{v} C^{*}(\Gamma) s_{v}$. By [18, Proposition 2.7] we have $p \precsim s_{v}$. Hence $p \precsim s_{v} \precsim h \precsim a$, so $p \precsim a$. As explained after [18, Proposition 2.6], there exists $x \in C^{*}(\Gamma)$ such that $p=x^{*} a x$. Let $y:=a^{1 / 2} x$. Then $y^{*} y=x^{*} a x=p$, and $q:=y y^{*}=a^{1 / 2} x x^{*} a^{1 / 2} \in B$. Since $p$ is infinite and Murray-von Neumann equivalent to $q$, we conclude that $q \in B$ is infinite.

The implication (v) $\Rightarrow$ (i) follows from [18, Proposition 4.7]. 
It now suffices to prove (ii) $\Rightarrow\left(\right.$ iii' $\left.^{\prime}\right) \Rightarrow\left(\right.$ iv' $\left.^{\prime}\right) \Rightarrow\left(\mathrm{v}^{\prime}\right) \Rightarrow(\mathrm{v})$. Recall that if $p$ is properly infinite and $\phi$ is a $C^{*}$-homomorphism such that $\phi(p) \neq 0$, then $\phi(p)$ is properly infinite. This and the second statement of Lemma 3.7 gives (ii) $\Rightarrow$ (iii'). The implication (iii') $\Rightarrow$ (iv') is trivial, and (iv') $\Rightarrow\left(v^{\prime}\right)$ follows from the argument for (iv) $\Rightarrow(v)$ above because any projection $p$ that is Murray-von Neumann equivalent to a properly infinite projection is itself is properly infinite. Since proper infiniteness is stronger than infiniteness, condition (v') implies (v), completing the proof.

Corollary 5.3. Let $\Lambda$ be a row-finite $k$-graph with no sources and suppose that $C^{*}(\Lambda)$ has real-rank zero. Then $C^{*}(\Lambda)$ is strongly purely infinite if and only if $s_{v}$ is properly infinite for every $v \in \Lambda^{0}$.

Proof. This follows from [31, Corollary 6.9] combined with and Lemmas 4.1 and 5.2.

Following [31], recall that a $C^{*}$-algebra $A$ has the ideal property, abbreviated (IP), if projections in $A$ separate ideals in $A$; that is, whenever $I, J$ are distinct ideals in $A$, there is a projection in $I \backslash J$.

Lemma 5.4. Let $\Lambda$ be a row-finite $k$-graph with no sources. Suppose that $\Lambda$ is strongly aperiodic. Then $C^{*}(\Lambda)$ has the ideal property.

Proof. Let $I, J$ be ideals of $C^{*}(\Lambda)$ such that $I \nsubseteq J$. Recall that for $H \subseteq \Lambda^{0}, I_{H}$ denotes the ideal in $C^{*}(\Lambda)$ generated by $\left\{s_{w}: w \in H\right\}$. By Corollary [3.9, $I=I_{H}$ and $J=I_{K}$ for some saturated hereditary $H, K \subseteq \Lambda^{0}$. Since $I \nsubseteq J$, we have $H \nsubseteq K$, say $v \in H \backslash K$. [32, Theorem 5.2(b)] shows that $H=\left\{w: s_{w} \in I_{H}\right\}$ and similarly for $K$, so we deduce that $s_{v}$ is a projection in $I_{H} \backslash I_{K}=I \backslash J$.

Remark 5.5. It not clear whether strong aperiodicity of $\Lambda$ is equivalent to property (IP) for $C^{*}(\Lambda)$. However, we obtain some easy partial results. First, suppose $C^{*}(\Lambda)$ is AF-so automatically has property (IP). By [11, Proposition 3.12], every ideal of $C^{*}(\Lambda)$ is gaugeinvariant, so Corollary 3.9, shows that $\Lambda$ is strongly aperiodic. Second, suppose that $C^{*}(\Lambda)$ has (IP) and is purely infinite. By [31, Proposition 2.11], $C^{*}(\Lambda)$ has topological dimension zero, so Corollary 3.9 shows that $\Lambda$ is strongly aperiodic.

Proof of Proposition 5.1. Since every vertex of $\Lambda$ has an aperiodic quartet, [16, Proposition 3.9] shows that $\Lambda$ is strongly aperiodic. Hence Lemma 5.4 shows that $C^{*}(\Lambda)$ has property (IP). By [31, Proposition 2.14], a $C^{*}$-algebra with property (IP) is purely infinite if and only if it is strongly purely infinite. So it suffices to prove that $C^{*}(\Lambda)$ is purely infinite.

To show that $C^{*}(\Lambda)$ is purely infinite it suffices to verify property (ii) of Lemma 5.2 , that is, it suffices to show that every $s_{v}$ is properly infinite.

Fix any $v \in \Lambda^{0}$. By assumption there exist $a, b \in \mathbb{Z}^{+}$, distinct $\alpha_{1}, \alpha_{2} \in v \Lambda^{a e_{1}} v$ and distinct $\beta_{1}, \beta_{2} \in v \Lambda^{b e_{2}} v$ such that $\beta_{2} \alpha_{1}=\alpha_{1} \beta_{2}, \beta_{2} \alpha_{2}=\alpha_{2} \beta_{2}, \beta_{1} \alpha_{1}=\alpha_{2} \beta_{1}$, and $\beta_{1} \alpha_{2}=$ $\alpha_{1} \beta_{1}$. By (CK3)-(CK4), for $i=1,2$ we have

$$
s_{v}=s_{\alpha_{i}}^{*} s_{\alpha_{i}}, \quad s_{\alpha_{i}} s_{\alpha_{i}}^{*} \leq \sum_{\lambda \in v \Gamma^{a e_{1}}} s_{\lambda} s_{\lambda}^{*}=s_{v} .
$$

Consequently, there exist distinct mutually orthogonal subprojections of $s_{v}$ in $C^{*}(\Lambda)$, each Murray-von Neumann equivalent to $s_{v}$. We conclude that $s_{v}$ is properly infinite. 


\section{EXAMPLES}

In this section we present three examples highlighting the necessity of the hypotheses in Theorem 4.3(1). All three examples are constructed using 2-graphs. Our result says that the combination of strong aperiodicity of $\Lambda$, injectivity of each $H_{1}\left(j^{H}\right)$, and pure infiniteness of $C^{*}(\Lambda)$ is sufficient to guarantee that $C^{*}(\Lambda)$ has real rank zero. Our three examples show that no combination of two of these conditions is strong enough.

We describe our examples using the 2-coloured graphs of [12]. A 2-coloured graph is a directed graph endowed with a map $c: E^{1} \rightarrow\left\{c_{1}, c_{2}\right\}$. We think of $c$ as determining a colour map from $E^{*}$ to the free abelian semigroup $\mathbb{F}_{2}$ generated by $\left\{c_{1}, c_{2}\right\}$ and for $w \in \mathbb{F}_{2}$, we say that $\lambda \in E^{*}$ is $w$-coloured if $c(\lambda)=w$. A collection of factorisation rules for $E$ is a range- and source-preserving bijection $\theta$ from the $c_{1} c_{2}$-coloured paths in $E^{*}$ to the $c_{2} c_{1}$-coloured paths. For $k=2$ the associativity condition of [12] is trivial, and so [12, Theorems 4.4 and 4.5] say that for every 2-coloured graph $(E, c)$ with a collection $\theta$ of factorisation rules, there is a unique 2-graph $\Lambda$ with $\Lambda^{e_{i}}=c^{-1}\left(c_{i}\right), \Lambda^{0}=E^{0}$, and ef $=f^{\prime} e^{\prime}$ in $\Lambda$ whenever $\theta(e f)=f^{\prime} e^{\prime}$ in $E^{*}$.

Example 6.1. Strong aperiodicity of $\Lambda$ (and hence topological dimension zero for $C^{*}(\Lambda)$ ) combined with strong pure infiniteness of $C^{*}(\Lambda)$ do not suffice for $C^{*}(\Lambda)$ to have real-rank zero, even in the special case $k=2$. To see this, fix $n \geq 3$ and let $(E, c)$ be the following 2-coloured graph:

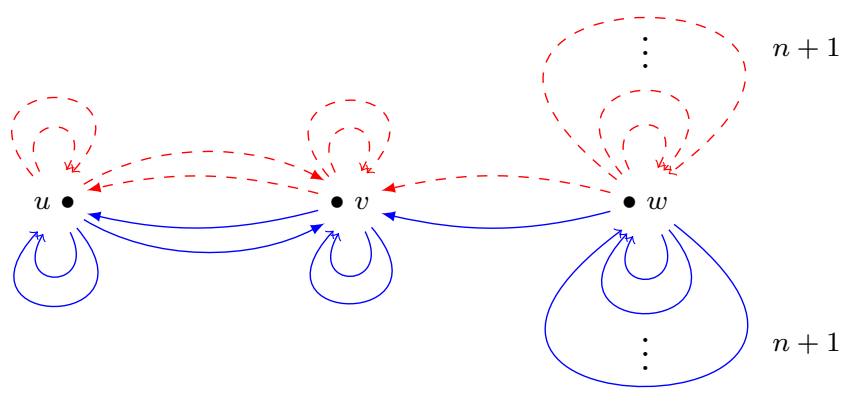

We define factorisation rules as follows. First, for each pair of vertices $x, y$ of $E$, list the blue edges in $x E^{1} y$ as $\left\{\alpha_{1}^{x, y}, \ldots, \alpha_{\left|x E^{1} y\right| / 2}^{x, y}\right\}$ and the red edges as $\left\{\beta_{1}^{x, y}, \ldots, \beta_{\left|x E^{1} y\right| / 2}^{x, y}\right\}$. When $x=y$, we write $\alpha_{i}^{x}$ and $\beta_{i}^{x}$ instead of $\alpha_{i}^{x, x}$ and $\beta_{i}^{x, x}$. For each vertex $x$, we define four factorisation rules by

$$
\beta_{2}^{x} \alpha_{1}^{x}=\alpha_{1}^{x} \beta_{2}^{x}, \quad \beta_{2}^{x} \alpha_{2}^{x}=\alpha_{2}^{x} \beta_{2}^{x}, \quad \beta_{1}^{x} \alpha_{1}^{x}=\alpha_{2}^{x} \beta_{1}^{x}, \quad \beta_{1}^{x} \alpha_{2}^{x}=\alpha_{1}^{x} \beta_{1}^{x} .
$$

We then specify $\alpha_{i}^{x, y} \beta_{j}^{y, z}=\beta_{i}^{x, y} \alpha_{j}^{y, z}$ for every blue-red path $\alpha_{i}^{x, y} \beta_{j}^{y, z}$ not appearing in the left-hand side of any of the factorisation rules in (6.1). With these factorisation rules we obtain a 2-graph $\Lambda$, which is row finite with no sources. The factorisation rules (6.1) yield a $((1,0),(0,1))$-aperiodic quartet at each vertex $x$ of $\Lambda$. Proposition 5.1 and [16, Proposition 3.9] ensures that $C^{*}(\Lambda)$ is strongly purely infinite and $\Lambda$ is strongly aperiodic for each $n \geq 1$. It is easy to check that $H:=\{w\}$ is a hereditary saturated subset of $\Lambda^{0}$. With the notation of (4.1) we have

$$
M_{i, H}^{t}=(n+1), \quad M_{i, H, \Lambda^{0} \backslash H}^{t}=\left(\begin{array}{ll}
0 & 1
\end{array}\right), \quad \text { and } \quad M_{i, \Lambda^{0} \backslash H}^{t}=\left(\begin{array}{ll}
2 & 1 \\
1 & 2
\end{array}\right)
$$


for each $i$. With $L_{2}:=\left\{M_{1, H, \Lambda^{0} \backslash H}^{t} b: b \in \mathbb{Z}\left(\Lambda^{0} \backslash H\right)\right.$ and $\left.\left(M_{1, \Lambda^{0} \backslash H}^{t}-1\right) b=0\right\}$ and $L_{1}:=\left\{\left(M_{1, H}^{t}-1\right) a: a \in \mathbb{Z} H\right\}$, we have $L_{1}=n \mathbb{Z}$ and $L_{2}=\mathbb{Z}$. So Proposition 4.4 implies that $H_{1}\left(j^{H}\right)$ is injective if and only if $n=1$. Hence Theorem 4.3(1) implies that $C^{*}(\Lambda)$ has real-rank zero for $n=1$, but not for $n>1$.

Example 6.2. The injectivity of each $H_{1}\left(j^{H}\right)$ combined with strong pure infiniteness of $C^{*}(\Lambda)$ do not suffice for $C^{*}(\Lambda)$ to have real-rank zero, even in the special case $k=2$. To see this, consider the following 2-coloured graph:

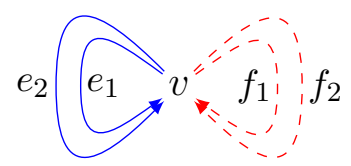

Define factorisation rules on $(E, c)$ by $e_{i} f_{j}=f_{i} e_{j}$, and let $\Lambda$ be the resulting 2-graph. By [21, Corollary 3.5(iii)], we have $C^{*}(\Lambda) \cong \mathcal{O}_{2} \otimes C(\mathbb{T})$. As $\mathcal{O}_{2}$ is purely infinite simple, $C^{*}(\Lambda)$ is strongly purely infinite, see [20, Theorem 1.3]. There are no non-trivial saturated hereditary subsets of $\Lambda^{0}$, so injectivity of each $H_{1}\left(j^{H}\right)$ is trivial. The $C^{*}$-algebra $C^{*}(\Lambda)$ is non-simple, but none of the proper ideals of $C^{*}(\Lambda)$ contain any vertex projections. Consequently, it follows from Corollary [3.9] that $\Lambda$ is not strongly aperiodic (alternatively, for each $\left.x \in v \Lambda^{\infty}, \sigma^{(1,-1)}(x)=x\right)$. Using Theorem 4.3 we conclude that $C^{*}(\Lambda)$ fails to have real-rank zero (cf. [31]).

Example 6.3. Strong aperiodicity combined with the injectivity of each $H_{1}\left(j^{H}\right)$ does not suffice for $C^{*}(\Lambda)$ to have real-rank zero, even in the special case $k=2$. To see this, consider the 2-coloured graph:

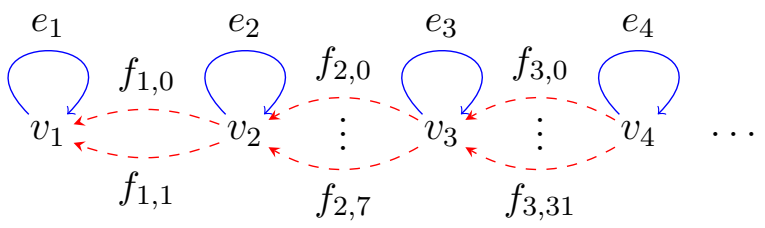

that has $2^{2 n-1}$ edges labelled $f_{n, 0}, \ldots, f_{n, 2^{2 n-1}-1}$ from $v_{n+1}$ to $v_{n}$ for each $n \geq 1$. There is a unique 2-graph for this 2-coloured graph with factorisation rules given by

$$
e_{n} f_{n, i}= \begin{cases}f_{n, i+1} e_{n+1} & \text { if } 0 \leq i<2^{n-1}-1 \\ f_{n, 0} e_{n+1} & \text { if } i=2^{n-1}-1 \\ f_{n, i} e_{n+1} & \text { if } 2^{n-1}<i<2^{2 n-1}\end{cases}
$$

so factorisation through a blue loop cyclicly permutes the first $2^{n-1}$ edges in $v_{n} \Lambda^{e_{2}} v_{n+1}$, and fixes the remaining ones.

For this example, in the notation of [26, Theorem 7.2], the $A_{n}$ are all nonzero $1 \times 1$ matrices, and each $\mathcal{F}_{n}^{1,1}$ is the permutation of $v_{n} \Lambda^{e_{2}} v_{n+1}$ induced by the factorisation rules. In particular, the constants $\beta_{1}$, as in [26, Definition 7.1], are given by

$$
\beta_{1}=\frac{1}{2^{2 n-1}} \cdot\left\{i: 2^{n-1}<i<2^{2 n-1}\right\},
$$

and so each $1-\beta_{1}\left(F_{n}^{1,1}\right)$ is $2^{n-1} / 2^{2 n-1}=2^{-n}$. So $\bar{\alpha}_{1}=\sum_{n=1}^{\infty}\left(1-\beta_{1}\left(\mathcal{F}_{n}^{1,1}\right)\right)=\sum 2^{-n}$ converges to $1<\infty$. Thus [26, Theorem 7.2(2)] implies that $C^{*}(\Lambda)$ has real-rank 1 . The graph $\Lambda$ is clearly cofinal. The numbers $\kappa\left(\mathcal{F}_{n}^{1,1}\right)$ denoting the maximal orders of elements 
of $v_{n} \Lambda^{e_{2}} v_{n+1}$ under $\mathcal{F}_{n}^{1,1}$ satisfy $\kappa\left(\mathcal{F}_{n}^{1,1}\right)=2^{n-1} \rightarrow \infty$. Hence the first statement of [26, Theorem 7.2] says that $C^{*}(\Lambda)$ is simple. It follows that $\Lambda$ is strongly aperiodic, and each $H_{1}\left(j^{H}\right)$ is injective because $H=\Lambda^{0}$ is the only non-empty saturated hereditary subset of $\Lambda^{0}$, in which case $H_{1}\left(j^{H}\right)$ is the identity map.

\section{REFERENCES}

[1] O. Bratteli and G.A. Elliott Structure spaces of approximately finite-dimensional C*-algebras. II, J. Funct. Anal. 30 (1978), 74-82.

[2] J. Brown, L.O. Clark, and A. Sierakowski, Purely infinite $C^{*}$-algebras associated to etale groupoids, Ergodic Theory Dynam. Systems 35 (2015), 2397-2411.

[3] L.G. Brown, On Higher Real and Stable Ranks for CCR $C^{*}$-algebras, preprint 2007 (preprint available at arXiv:0708.3072).

[4] L.G. Brown and G.K. Pedersen, $C^{*}$-algebras of real rank zero, J. Funct. Anal. 99 (1991), 131-149.

[5] L.G. Brown and G.K. Pedersen, Limits and $C^{*}$-algebras of low rank or dimension, J. Operator Theory 61 (2009), 381-417.

[6] T.M. Carlsen, S. Kang, J. Shotwell, and A. Sims, The primitive ideals of the Cuntz-Krieger algebra of a row-finite higher-rank graph with no sources, J. Funct. Anal. 266 (2014), 2570-2589.

[7] A. Connes, Noncommutative geometry, Academic Press, Inc., San Diego, CA, 1994. ISBN: 0-12185860-X.

[8] J. Dixmier, $C^{*}$-algebras, Translated from the French by Francis Jellett. North-Holland Mathematical Library, Vol. 15. North-Holland Publishing Co., Amsterdam-New York-Oxford, 1977. xiii +492 pp. ISBN: 0-7204-0762-1

[9] G.A. Elliott and D.E. Evans, The structure of the irrational rotation $C^{*}$-algebra, Ann. of Math. (2) 138 (1993), 477-501.

[10] D.G. Evans, On the K-theory of higher rank graph $C^{*}$-algebras, New York J. Math. 14 (2008), 1-31.

[11] D.G. Evans and A. Sims, When is the Cuntz-Krieger algebra of a higher-rank graph approximately finite-dimensional?, J. Funct. Anal. 263 (2012), 183-215.

[12] R. Hazlewood, I. Raeburn, A. Sims, and S.B.G. Webster, Remarks on some fundamental results about higher-rank graphs and their $C^{*}$-algebras, Proc. Edinb. Math. Soc. (2) 14 (2013), 575-597.

[13] J.H. Hong and W. Szymanski, Purely infinite Cuntz-Krieger algebras of directed graphs, Bull. Lond. Math. Soc. (5) 35 (2003), 689-696.

[14] J.A. Jeong, G.H. Park, and D.Y. Shin Stable rank and real rank of graph $C^{*}$-algebras, Pacific J. Math. 200 (2001), 331-343.

[15] J.A. Jeong and G.H. Park. Graph $C^{*}$-algebras with real rank zero, J. Funct. Anal. 188 (2002), 216-226.

[16] S. Kang and D. Pask, Aperiodic and primitive ideals of row-finite k-graphs, Int. J. Math. 25 (2014), 1450022 [25 pages].

[17] E. Kirchberg, Das nicht-kommutative Michael-Auswahlprinzip und die Klassifikation nicht-einfacher Algebren, $C^{*}$-algebras (Münster, 1999), Springer, Berlin, 2000, pp. 92-141.

[18] E. Kirchberg and M. Rørdam, Non-simple purely infinite $C^{*}$-algebras, Amer. J. Math. 122 (2000), 637-666.

[19] E. Kirchberg and M. Rørdam, Infinite non-simple $C^{*}$-algebras: absorbing the Cuntz algebras $\mathcal{O}_{\infty}$, Adv. Math. 167 (2002), 195-264.

[20] E. Kirchberg and A. Sierakowski, Filling families and strong pure infiniteness, preprint, 2014.

[21] A. Kumjian and D. Pask, Higher rank graph $C^{*}$-algebras, New York J. Math. 6 (2000), 1-20.

[22] A. Kumjian, D. Pask, I. Raeburn, and J. Renault, Graphs, groupoids, and Cuntz-Krieger algebras, J. Funct. Anal. 144 (1997), 505-541.

[23] A. Kumjian, D. Pask, and A. Sims, $C^{*}$-algebras associated to coverings of k-graphs, Doc. Math. 13 (2008), 161-205.

[24] A. Kumjian, D. Pask, and A. Sims, On the K-theory of twisted higher-rank-graph $C^{*}$-algebras, J. Math. Anal. Appl., 401 (2013), 104-113.

[25] D. Pask, J. Quigg, and I. Raeburn, Coverings of k-graphs, J. Algebra 289 (2005), 161-191. 
[26] D. Pask, I. Raeburn, M. Rørdam and A. Sims Rank-two graphs whose $C^{*}$-algebras are direct limits of circle algebras, J. Funct. Anal. 239 (2006), 137-178.

[27] C. Pasnicu, Extensions of AH algebras with the ideal property, Proc. Edinburgh Math. Soc. (2) 42 (1999), 65-76.

[28] C. Pasnicu, The ideal property, the projection property, continuous fields and crossed products, J. Math. Anal. Appl. 323 (2006), 1213-1224.

[29] C. Pasnicu, Real rank zero and continuous fields of $C^{*}$-algebras, Bull. Math. Soc. Sci. Math. Roumanie (N.S.) 48 (2005), 319-325.

[30] C. Pasnicu, and N.C. Phillips Permanence properties for crossed products and fixed point algebras of finite groups., Trans. Amer. Math. Soc. 366 (2014), 4625-4648.

[31] C. Pasnicu and M. Rørdam, Purely infinite $C^{*}$-algebras of real rank zero, J. reine angew. Math. 613 (2007), 51-73.

[32] I. Raeburn, A. Sims, and T. Yeend, Higher-rank graphs and their $C^{*}$-algebras, Proc. Edinb. Math. Soc. (2) 46 (2003), 99-115.

[33] J. Renault, A groupoid approach to $C^{*}$-algebras, Lecture Notes in Mathematics, 793. Springer, Berlin, 1980. ISBN: 3-540-09977-8.

[34] J. Renault, Examples of masas in $C^{*}$-algebras, Operator structures and dynamical systems, 259-265, Contemp. Math., 503, Amer. Math. Soc., Providence, RI, 2009.

[35] L. Robert and A. Tikuisis, Nuclear dimension and Z-stability of non-simple $C^{*}$-algebras, Trans. Amer. Math. Soc., to appear (preprint available at arXiv:1308.2941).

[36] D. Robertson and A. Sims, Simplicity of $C^{*}$-algebras associated to higher-rank graphs., Bull. Lond. Math. Soc. (2) 39 (2007), 337-344.

[37] A. Sims, Gauge-invariant ideals in the $C^{*}$-algebras of finitely aligned higher-rank graphs, Canad. J. Math. 58 (2006), 1268-1290.

[38] H. Thiel The topological dimension of type I $C^{*}$-algebras, Operator algebra and dynamics, 305-328, Springer Proc. Math. Stat., 58, Springer, Heidelberg, 2013.

[39] D.P. Williams, Crossed Products of $C^{*}$-algebra, Mathematical Surveys and Monographs, 134. Amer. Math. Soc., Providence, RI, 2007. ISBN: 978-0-8218-4242-3.

[40] S. Zhang, Certain $C^{*}$-algebras with real rank zero and their corona and multiplier algebras, Part I, Pacific J. Math. 155 (1992), 169-197.

E-mail address: dpask/asierako/asims@uow.edu.au

(D. Pask, A. Sierakowski and A. Sims) School of Mathematics And Applied Statistics, UniVERsity of Wollongong, Wollongong NSW 2522, AUSTRALIA 